ICASE Report No. 96-52

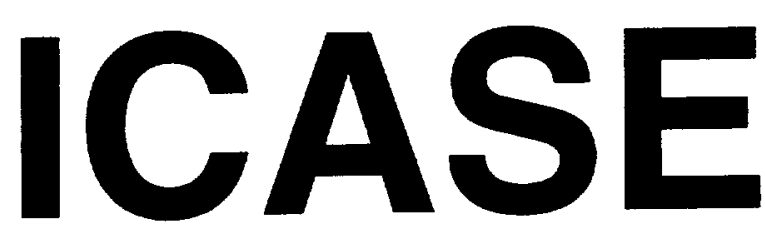

\title{
TRANSPORT COEFFICIENTS IN WEAKLY COMPRESSIBLE TURBULENCE
}

\section{Robert Rubinstein Gordon Erlebacher}

NASA Contract No. NAS1-19480

August 1996

Institute for Computer Applications in Science and Engineering

NASA Langley Research Center

Hampton, VA 23681-0001

Operated by Universities Space Research Association

NEA

National Aeronautics and

Space Administration

Langley Research Center

Hampton, Virginia 23681-0001 



\title{
TRANSPORT COEFFICIENTS IN WEAKLY COMPRESSIBLE TURBULENCE
}

\author{
Robert Rubinstein and Gordon Erlebacher* \\ Institute for Computer Applications in Science and Engineering \\ NASA Langley Research Center \\ Hampton, VA 23681
}

\begin{abstract}
A theory of transport coefficients in weakly compressible turbulence is derived by applying Yoshizawa's two-scale direct interaction approximation to the compressible equations of motion linearized about a state of incompressible turbulence. The result is a generalization of the eddy viscosity representation of incompressible turbulence. In addition to the usual incompressible eddy viscosity, the calculation generates eddy diffusivities for entropy and pressure, and an effective bulk viscosity acting on the mean flow. The compressible fluctuations also generate an effective turbulent mean pressure and corrections to the speed of sound. Finally, a prediction unique to Yoshizawa's two-scale approximation is that terms containing gradients of incompressible turbulence quantities also appear in the mean flow equations. The form these terms take is described.
\end{abstract}

* This research was supported by the National Aeronautics and Space Administration under NASA Contract No. NAS1-19480 while the authors were in residence at the Institute for Computer Applications in Science and Engineering (ICASE), NASA Langley Research Center, Hampton, VA 23681-0001. 



\section{Introduction}

This paper derives a gradient transport model for weakly compressible turbulence which generalizes the eddy viscosity description of incompressible turbulence. The model is derived by applying Yoshizawa's two-scale direct interaction approximation (TSDIA) ${ }^{1}$ to the theory of weakly compressible turbulence. In this theory, the compressible field quantities are analyzed by linearization about a state of incompressible turbulence. ${ }^{2,3}$

The present model can be compared to Yoshizawa's theory, ${ }^{4}$ the first comprehensive attempt to derive a general transport model for compressible turbulence. Like Yoshizawa's, the present theory requires the derivation of a direct interaction approximation ${ }^{5}$ (DIA) for compressible turbulence. Whereas Yoshizawa's theory allows strong compressibility effects, the restriction in the present work to weak compressibility makes possible a somewhat more complete treatment of the DIA response functions and leads to more explicit expressions for the transport coefficients. In particular, the present theory treats the complete matrix of response functions, including various coupling terms which produce the wave motions characteristic of compressible flow.

The DIA for weakly compressible turbulence derived here can be compared to theories proposed by Bertoglio et al. ${ }^{6}$ Like them, we derive approximate formulas for the response functions; although a comprehensive DIA theory of compressible turbulence was formulated by Hartke et al, ${ }^{\top}$ explicit expressions for the DIA field descriptors were not given. Renormalization group methods were applied to strongly compressible turbulence by Staroselski et al. ${ }^{8}$ The spectral dynamics of weakly compressible turbulence has also been modeled using EDQNM by Bertoglio et $\mathrm{al}^{6}$ and by Bataille et al. ${ }^{9}$

The theory of weakly compressible turbulence has been considered from the TSDIA viewpoint by Shimomura; ${ }^{10}$ however, this work had the quite different goal of a more refined treatment of buoyancy effects than is possible in the Boussinesq approximation. Another refinement of the Boussinesq equations through low Mach number expansions is investigated by Mlaouah et al. ${ }^{11}$ A distinct theory of weak compressibility has been proposed by Girimaji. ${ }^{12}$ In this theory, the compressible part of the velocity field is decomposed into pressure and temperature-dominated components. Any of these theories could have been used as a starting point for TSDIA instead of the straightforward linearization adopted here. 
A more traditional approach to modeling compressible turbulence generalizes incompressible models through mass-weighted averaging; a definitive survey of this viewpoint has been given by Huang et al. ${ }^{13}$ Like Yoshizawa, we attempt instead to derive a model from a dynamic theory of compressible turbulence. Ristorcelli ${ }^{14}$ has proposed a pseudosound theory of compressible turbulence for compact flows of engineering interest. In this weakly compressible theory the length scales of the acoustic fluctuations scale as the inverse Mach number with respect to the vortical length scales. This allows the neglect of wave propagation effects.

The present model is derived from a three-component decomposition of the compressible flow field: after the usual Reynolds decomposition into mean and fluctuating components, the fluctuations are further decomposed following Zank and Matthaeus ${ }^{2}$ and Erlebacher et $\mathrm{al}^{3}$ into an incompressible field and small compressible perturbations. The compressible field is treated by linearization about the incompressible field; it follows that this theory only applies to compressible turbulence at small turbulent Mach number.

As usual, the Reynolds averaging introduces various correlations of fluctuating quantities which require closure; whereas for incompressible turbulence, only the Reynolds stress arises this way, the increased number of field quantities describing compressible turbulence naturally generates a larger number of such correlations. These unknown correlations are all closed in terms of gradients of the mean fields and single point descriptors of the incompressible field using Yoshizawa's TSDIA formalism. ${ }^{1}$ Unlike Yoshizawa's theory ${ }^{4}$ of compressible turbulence, which introduces the density variance as a new descriptor of strongly compressible turbulence, the present linearized theory only requires single point descriptors of incompressible turbulence. These are chosen following the usual two equation models as turbulence kinetic energy $K$ and dissipation rate $\varepsilon$.

As Yoshizawa has demonstrated, ${ }^{1}$ the TSDIA really generates an infinite series for the unknown correlations both in powers of the mean gradients and in the order of differentiation of the mean fields. The present analysis is limited to the terms of lowest order in this expansion: those which are linear in the mean fields and which contain spatial derivatives of order two at most. A large number of potentially interesting nonlinear effects, analogous to the normal stress effects in simple incompressible shear flow, could be described by computing the higher order terms in the series. 
In addition to the usual incompressible eddy viscosity, the calculation generates eddy diffusivities for entropy and pressure and an effective bulk viscosity acting on the mean flow. The compressible fluctuations also generate an effective turbulent mean pressure and corrections to the speed of sound. The "renormalized" bulk viscosity, mean pressure, and sound speed are consequences of coupling among the response equations which correspond to the generation of sound waves in compressible turbulence. These effects would be suppressed by a "diagonal" approximation which ignores such couplings.

These effective properties have the character of enhancements of molecular properties by turbulent fluctuations. But a prediction that appears to be unique to TSDIA is that terms containing gradients of turbulence quantities also appear in the mean flow equations. Because of the large number of such terms and their complexity, only a preliminary account of the effect of these terms on single point modeling is given. Explicit evaluation of these terms is reserved for future work.

It should be stressed that the present model only attempts to close the correlations which arise from Reynolds averaging of the equations of motion. The effects of compressibility on the two-equation model ${ }^{14,15}$ are not addressed, although we indicate later how these effects could be analyzed within the present setting.

An important question concerns the validity of linearized theories of compressible turbulence at asymptotically large times: ${ }^{16}$ in the infinite time limit, the compressible field might build up to amplitude levels sufficient to invalidate a linearized theory. For example, Staroselski et $\mathrm{al}^{8}$ argue that equipartition between compressible and incompressible fluctuations is a possible outcome of the long time evolution of compressible turbulence. But when the compressible field is generated entirely from the incompressible field, the weakness of the relevant energy transfer mechanism may permit a range of times, suffciently large for many applications, over which the linearization remains valid. Numerical simulations of energy transfer in weakly compressible turbulence by Bataille et al ${ }^{17}$ provide examples of this possibility.

\section{Formulation of the theory of weakly compressible turbulence}

The inviscid governing equations are taken in the form

$$
\frac{\partial s}{\partial t}+\mathbf{u} \cdot \nabla s=0
$$




$$
\begin{aligned}
& \frac{\partial \mathbf{u}}{\partial t}+\mathbf{u} \cdot \nabla \mathbf{u}+\frac{1}{\rho} \nabla p=0 \\
& \frac{\partial p}{\partial t}+\mathbf{u} \cdot \nabla p+\gamma p \nabla \cdot \mathbf{u}=0
\end{aligned}
$$

with dependent variables entropy $s$, velocity $\mathbf{u}$, and pressure $p$. Density can be evaluated from entropy and pressure through the thermodynamic relation

$$
s / C_{V}=\log \frac{p}{p_{0}}-\gamma \log \frac{\rho}{\rho_{0}}
$$

In what follows, $s$ denotes entropy divided by $C_{V}$ to simplify the notation.

The analysis is based on a three-component decomposition of the velocity field into mean quantities, incompressible fluctuations, and small compressible perturbations. Accordingly, first introduce the usual Reynolds decomposition of the fields $s, \mathbf{u}, p$ into mean and fluctuations and then further decompose the fluctuations into an incompressible field and compressible perturbations following Zank and Matthaeus. ${ }^{2}$ Thus, the field quantities are written as

$$
\begin{aligned}
& s=S+s^{\prime} \\
& \mathbf{u}=\mathbf{U}+\mathbf{u}^{\infty}+\mathbf{u}^{\prime} \\
& p=P+p^{\infty}+p^{\prime}
\end{aligned}
$$

where $S, \mathbf{U}, P$ denote the mean entropy,velocity, and pressure and $\mathbf{u}^{\infty}, p^{\infty}$ the incompressible velocity and pressure fluctuations. Note that the field $\mathbf{U}$ need not be solenoidal. Write the density as

$$
\rho=R+\rho^{\prime}
$$

The density fluctuation $\rho^{\prime}$ can be evaluated by linearizing Eq. (2) as

$$
\rho^{\prime}=c^{-2}\left(p^{\infty}+p^{\prime}\right)-\gamma^{-1} R s^{\prime}
$$

where the mean speed of sound is defined by the thermodynamic relation

$$
c^{2}=\gamma \frac{P}{R}
$$

The compressible velocity perturbation $\mathbf{u}^{\prime}$ need not be irrotational; in fact, the interaction of the compressible field with the base isotropic incompressible field $\mathbf{u}^{\infty}$ generates additional solenoidal motions. The dynamics of the compressible perturbations $s^{\prime}, \mathbf{u}^{\prime}, p^{\prime}$ will 
be analyzed by linearization about the mean fields and incompressible fluctuations: this defines the theory of weakly compressible turbulence.

The mean equations are obtained by substituting the decomposition Eq. (3) into Eq. (1), dropping terms which are nonlinear in the compressible quantities, and averaging. Then

$$
\begin{aligned}
& \frac{\partial S}{\partial t}+\mathbf{U} \cdot \nabla S=-\nabla \cdot\left\langle\mathbf{u}^{\infty} s^{\prime}\right\rangle \\
& \left.R\left(\frac{\partial \mathbf{U}}{\partial t}+\mathbf{U} \cdot \nabla \mathbf{U}\right)+\nabla P=-R \nabla \cdot\left\langle\mathbf{u}^{\infty} \mathbf{u}^{\infty}+\mathbf{u}^{\infty} \mathbf{u}^{\prime}\right\rangle-R<\mathbf{u}^{\prime} \cdot \nabla \mathbf{u}^{\infty}\right\rangle \\
& \left.\quad+\frac{1}{R c^{2}} \nabla<\frac{1}{2} p^{\infty} p^{\infty}+p^{\infty} p^{\prime}\right\rangle-\frac{1}{\gamma}\left\langle s^{\prime} \nabla p^{\infty}\right\rangle \\
& \frac{\partial P}{\partial t}+\mathbf{U} \cdot \nabla P+\gamma P \nabla \cdot \mathbf{U}=-\nabla \cdot\left\langle\mathbf{u}^{\infty} p^{\infty}+\mathbf{u}^{\infty} p^{\prime}+\gamma \mathbf{u}^{\prime} p^{\infty}\right\rangle \\
& \quad+(\gamma-1)<\mathbf{u}^{\prime} \cdot \nabla p^{\infty}>
\end{aligned}
$$

where in Eq. ( 7$), \rho^{\prime}$ has been eliminated in favor of $p^{\infty}, p^{\prime}$, and $s^{\prime}$ using Eq. (5). The derivation of Eq. (7) requires the expansion of the ratio $1 /\left(R+\rho^{\prime}\right)$ in powers of $\rho^{\prime} / R$. Following the usual practice in the theory of compressible turbulence, only the term linear in $\rho^{\prime}$ has been retained. Computing this series to higher order in $\rho^{\prime}$ would generate a series in powers of $p^{\infty} / c^{2}$. Although linearization does not justify ignoring these terms, they generate a series in powers of the turbulent Mach number which can reasonably be truncated at lowest order.

The incompressible field satisfies

$$
\begin{aligned}
& \frac{\partial \mathbf{u}^{\infty}}{\partial t}+\mathbf{u}^{\infty} \cdot \nabla \mathbf{u}^{\infty}+\frac{1}{R} \nabla p^{\infty}=-\left[\mathbf{U} \cdot \nabla \mathbf{u}^{\infty}+\mathbf{u}^{\infty} \cdot(\nabla \mathbf{U})^{S}\right]+\left\langle\mathbf{u}^{\infty} \cdot \nabla \mathbf{u}^{\infty}\right\rangle \\
& \nabla \cdot \mathbf{u}^{\infty}=0
\end{aligned}
$$

Eqs. (9) and (10) require that the incompressible pressure $p^{\infty}$ satisfy the Poisson equation

$$
\nabla \cdot \frac{1}{R} \nabla p^{\infty}=-\nabla \cdot\left(\mathbf{u}^{\infty} \cdot \nabla \mathbf{u}^{\infty}\right)-\nabla \cdot\left[\mathbf{U} \cdot \nabla \mathbf{u}^{\infty}+\mathbf{u}^{\infty} \cdot(\nabla \mathbf{U})^{S}+\left\langle\mathbf{u}^{\infty} \cdot \nabla \mathbf{u}^{\infty}\right\rangle\right]
$$

These equations have been investigated in Yoshizawa's TSDIA analysis of incompressible turbulence; ${ }^{1}$ the results of that analysis will be assumed in what follows. The traceless part of the mean velocity gradient appears in the incompressible momentum equation Eq. (9); it is defined by the decomposition

$$
\nabla U=(\nabla U)^{S}+(\nabla U)^{C}
$$


in which

$$
\begin{aligned}
(\nabla U)_{i j}^{S} & =\frac{\partial U_{i}}{\partial x_{j}}-\frac{1}{3} \frac{\partial U_{p}}{\partial x_{p}} \delta_{i j} \\
(\nabla U)_{i j}^{C} & =\frac{1}{3} \frac{\partial U_{p}}{\partial x_{p}} \delta_{i j}
\end{aligned}
$$

The component $(\nabla U)^{C}$ is added to the compressible momentum equation below.

The derivation of the linearized equations for the compressible fluctuations is routine and leads to

$$
\begin{aligned}
& \frac{\partial s^{\prime}}{\partial t}+\mathbf{u}^{\infty} \cdot \nabla s^{\prime}+C^{s}=0 \\
& \frac{\partial \mathbf{u}^{\prime}}{\partial t}+\mathbf{u}^{\infty} \cdot \nabla \mathbf{u}^{\prime}+\mathbf{u}^{\prime} \cdot \nabla \mathbf{u}^{\infty}+\frac{1}{R} \nabla p^{\prime}-\frac{p^{\prime}}{c^{2} R^{2}} \nabla p^{\infty}+\frac{1}{\gamma} R s^{\prime} \nabla p^{\infty}+\mathbf{C}^{u}=0 \\
& \frac{\partial p^{\prime}}{\partial t}+\mathbf{u}^{\infty} \cdot \nabla p^{\prime}+R c^{2} \nabla \cdot \mathbf{u}^{\prime}+\mathbf{u}^{\prime} \cdot \nabla p^{\infty}+\gamma p^{\infty} \nabla \cdot \mathbf{u}^{\prime}+C^{p}=-p^{\dagger}
\end{aligned}
$$

where the source term in Eq. (13) is the incompressible quantity

$$
p^{\dagger}=\frac{\partial p^{\infty}}{\partial t}+\mathbf{u}^{\infty} \cdot \nabla p^{\infty}
$$

and the terms coupling the fluctuating and mean fields in Eqs. (11)-(13) are

$$
\begin{aligned}
C^{s} & =\mathbf{U} \cdot \nabla s^{\prime}+\mathbf{u}^{\infty} \cdot \nabla S+\mathbf{u}^{\prime} \cdot \nabla S-<\mathbf{u}^{\infty} \cdot \nabla s^{\prime}> \\
\mathbf{C}^{u} & =\mathbf{U} \cdot \nabla \mathbf{u}^{\prime}+\mathbf{u}^{\prime} \cdot \nabla \mathbf{U}-\frac{\rho^{\prime}}{R^{2}} \nabla P-\frac{\rho^{\prime}}{R^{2}} \nabla p^{\prime}-\frac{1}{R^{2} c^{2}} p^{\infty} \nabla p^{\infty} \\
& +\mathbf{u}^{\infty} \cdot(\nabla \mathbf{U})^{C}-<\mathbf{u}^{\infty} \cdot \nabla \mathbf{u}^{\prime}+\mathbf{u}^{\prime} \nabla \mathbf{u}^{\infty}-\frac{1}{R^{2}} \rho^{\prime} \nabla p^{\infty}-\frac{1}{R^{2}} \rho^{\prime} \nabla p^{\prime}> \\
C^{p} & =\mathbf{U} \cdot \nabla p^{\infty}+\mathbf{U} \cdot \nabla p^{\prime}+\mathbf{u}^{\infty} \cdot \nabla P+\gamma p^{\infty} \nabla \cdot \mathbf{U}+\mathbf{u}^{\prime} \cdot \nabla P \\
& +\gamma p^{\prime} \nabla \cdot \mathbf{U}-<\mathbf{u}^{\infty} \cdot \nabla p^{\infty}+\mathbf{u}^{\infty} \cdot \nabla p^{\prime}+\mathbf{u}^{\prime} \cdot \nabla p^{\infty}+\gamma p^{\infty} \nabla \cdot \mathbf{u}^{\prime}>
\end{aligned}
$$

In Eq. (16), the density fluctuation must be expressed in terms of entropy and pressure by Eq. (5).

\section{DIA analysis of homogeneous isotropic weakly compressible turbulence}

Analytical theories of turbulence such as $\mathrm{DIA}^{5}$ provide a systematic procedure for evaluating the correlations generated by Reynolds averaging. Although DIA can be formulated even for arbitrarily inhomogeneous and anisotropic turbulence, ${ }^{18}$ the resulting 
theory proves to be intractably complex. TSDIA provides a useful approximation. Heuristically, it computes perturbatively about an isotropic and locally homogeneous state of turbulence which is described to lowest order by DIA. The effects of coupling to mean fields are evaluated by a perturbation series in a scale ratio parameter. This perturbative treatment restricts TSDIA to weakly inhomogeneous and anisotropic turbulence; however, since universal single-point modeling of turbulence with arbitrarily strong inhomogeneity and anisotropy is impossible in principle, this restriction does not unduly limit the applicability of TSDIA to turbulence modeling.

Analytically, the local homogeneity of the lowest order TSDIA field means that moments of this field at each point are evaluated using DIA results for homogeneous isotropic turbulence generalized for weak inhomogeneity by allowing all single point descriptors to vary slowly with space and time. This approximation, justified in the formal development of TSDIA, ${ }^{1}$ occurs in many physical theories, for example in thermodynamics, when a thermodynamic system is taken to have a temporally or spatially variable temperature.

In the present problem, Eqs. (11)-(13) describe a coupled system of passive fields. The application of DIA to this problem is not straightforward because the coupling between the compressible and incompressible fields cannot be analyzed exactly. Approximations are required which are both analytically tractable and preserve the important physical properties of the problem. Further discussion appears in Appendix I.

The simplest suitable approximation is obtained by treating both the mean field couplings and the terms which depend on the incompressible pressure as perturbations. Thus, write Eqs. (11)-(13) as

$$
\begin{aligned}
& \frac{\partial s}{\partial t}+\mathbf{u}^{\infty} \cdot \nabla s=-C^{s} \\
& \frac{\partial \mathbf{u}}{\partial t}+\mathbf{u}^{\infty} \cdot \nabla \mathbf{u}+\mathbf{u} \cdot \nabla \mathbf{u}^{\infty}+\frac{1}{\rho_{0}} \nabla p=\left(\rho / \rho_{0}^{2}\right) \nabla p^{\infty}-\mathbf{C}^{u} \\
& \frac{\partial p}{\partial t}+\mathbf{u}^{\infty} \cdot \nabla p+\rho_{0} c_{0}^{2} \nabla \cdot \mathbf{u}=-p^{\dagger}-\left(\mathbf{u} \cdot \nabla p^{\infty}+\gamma p^{\infty} \nabla \cdot \mathbf{u}\right)-C^{p}
\end{aligned}
$$

In Eqs. (18)-(20), the primes on compressible field quantities have been dropped. Since we are treating the homogeneous problem, $R=\rho_{0}$ and $c=c_{0}$ are assumed constant in Eqs. (18)-(20). Following the perturbation scheme outlined in Appendix I, we must evaluate 
the response matrix of the homogeneous system

$$
\begin{aligned}
& \frac{\partial s}{\partial t}+\mathbf{u}^{\infty} \cdot \nabla s=0 \\
& \frac{\partial \mathbf{u}}{\partial t}+\mathbf{u}^{\infty} \cdot \nabla \mathbf{u}+\mathbf{u} \cdot \nabla \mathbf{u}^{\infty}+\frac{1}{\rho_{0}} \nabla p=0 \\
& \frac{\partial p}{\partial t}+\mathbf{u}^{\infty} \cdot \nabla p+\rho_{0} c_{0}^{2} \nabla \cdot \mathbf{u}=0
\end{aligned}
$$

and treat all terms on the right side of Eqs. (18)-(20) perturbatively. The corresponding homogeneous form of the incompressible equations are

$$
\begin{aligned}
& \frac{\partial \mathbf{u}^{\infty}}{\partial t}+\mathbf{u}^{\infty} \cdot \nabla \mathbf{u}^{\infty}+\frac{1}{\rho_{0}} \nabla p^{\infty}=0 \\
& \nabla \cdot \mathbf{u}^{\infty}=0
\end{aligned}
$$

and as usual, these equations imply that the pressure satisfies

$$
\nabla^{2} p^{\infty}=-\rho_{0} \nabla \cdot\left(\mathbf{u}^{\infty} \cdot \nabla \mathbf{u}^{\infty}\right)
$$

Note that the $s$ equation Eq. (21) decouples from the others: $s$ is a passive scalar advected by the incompressible velocity field $\mathbf{u}^{\infty}$. The response matrix therefore has the structure

$$
\mathbf{G}=\left[\begin{array}{ccc}
G^{s s} & 0 & 0 \\
0 & G_{i j}^{u u} & G_{j}^{u p} \\
0 & G_{i}^{p u} & G^{p p}
\end{array}\right]
$$

The elements of the response matrix are defined $a s^{5}$

$$
\begin{aligned}
& G^{s s}=<\delta s / \delta f^{s}> \\
& G_{i j}^{u u}=<\delta u_{i} / \delta f_{j}^{u}> \\
& G_{i}^{u p}=<\delta u_{i} / \delta f^{p}> \\
& G_{j}^{p u}=<\delta p / \delta f_{j}^{u}> \\
& G^{p p}=<\delta p / \delta f^{p}>
\end{aligned}
$$

where the quantities $f^{s}, f_{j}^{u}, f^{p}$ are small perturbations added respectively to Eqs. (21)(23).

The spatial homogeneity of the fields justifies Fourier transformation in space. Since $s$ is a passive scalar, its response function $G^{s s}$ satisfies the standard DIA equation ${ }^{19}$

$$
\frac{\partial G^{s s}}{\partial t}(\mathbf{k}, t, r)+\int_{r}^{t} d s \eta^{s s}(\mathbf{k}, t, s) G^{s s}(\mathbf{k}, s, r)=\delta(t-r)
$$


where the damping factor $\eta^{s s}$ in Eq. (25) is evaluated as

$$
\eta^{s s}(\mathbf{k}, t, r)=k_{m} k_{n} \int_{\mathbf{k}=\mathbf{p}+\mathbf{q}} d \mathbf{p} d \mathbf{q} G^{s s}(\mathbf{p}, t, r) Q_{m n}(\mathbf{q}, t, r)
$$

In Eq. (26), $Q_{m n}$ is the incompressible two time correlation function

$$
<\mathbf{u}_{m}^{\infty}(\mathbf{k}, t) \mathbf{u}_{n}^{\infty}\left(\mathbf{k}^{\prime}, r\right)>=Q_{m n}(\mathbf{k}, t, r) \delta\left(\mathbf{k}+\mathbf{k}^{\prime}\right)
$$

The $\delta$ function forcing in Eq. (25) summarizes the initial and causality conditions for $G^{s s}$ which are

$$
\begin{aligned}
& G^{s s}(\mathbf{k}, t, r)=0 \text { for } t<r \\
& G^{s s}(\mathbf{k}, t, t)=1
\end{aligned}
$$

The spatial Fourier transforms of Eqs. (22) and (23) are

$$
\begin{gathered}
\frac{\partial u_{i}}{\partial t}(\mathbf{k}, t)+\frac{1}{\rho_{0}} i k_{i} p(\mathbf{k}, t)+\int_{\mathbf{k}=\mathbf{p}+\mathbf{q}} d \mathbf{p} d \mathbf{q} \Gamma_{i m n}^{1}(\mathbf{k}, \mathbf{p}, \mathbf{q}) u_{m}^{\infty}(\mathbf{p}, t) u_{n}(\mathbf{q}, t)=0 \\
\frac{\partial p}{\partial t}(\mathbf{k}, t)+i \rho_{0} c^{2} k_{i} u_{i}(\mathbf{k}, t)+\int_{\mathbf{k}=\mathbf{p}+\mathbf{q}} d \mathbf{p} d \mathbf{q} \Gamma_{n}^{2}(\mathbf{k}, \mathbf{p}, \mathbf{q}) u_{n}^{\infty}(\mathbf{p}, t) p(\mathbf{q}, t)=0
\end{gathered}
$$

The couplings between the compressible and incompressible fields in Eq. (27) are defined by

$$
\begin{aligned}
\Gamma_{i m n}^{1}(\mathbf{k}, \mathbf{p}, \mathbf{q}) & =i\left(q_{m} \delta_{i n}+p_{n} \delta_{i m}\right) \\
\Gamma_{n}^{2}(\mathbf{k}, \mathbf{p}, \mathbf{q}) & =i k_{n}
\end{aligned}
$$

The DIA equations for the response functions are easily shown to be

$$
\begin{aligned}
& \dot{G}_{i j}^{u u}+\frac{1}{\rho_{0}} i k_{i} G_{j}^{p u}+\eta_{i}^{u p} * G_{j}^{p u}+\eta_{i s}^{u u} * G_{s j}^{u u}=\delta(t-r) \\
& \dot{G}_{i}^{u p}+\frac{1}{\rho_{0}} i k_{i} G^{p p}+\eta_{i}^{u p} * G^{p p}+\eta_{i s}^{u u} * G_{s}^{u p}=0 \\
& \dot{G}_{i}^{p u}+i \rho_{0} c^{2} k_{j} G_{j i}^{u u}+\eta_{j}^{p u} * G_{j i}^{u u}+\eta^{p p} * G_{i}^{p u}=0 \\
& \dot{G}^{p p}+i \rho_{0} c^{2} k_{j} G_{j}^{u p}+\eta_{j}^{p u} * G_{j}^{u p}+\eta^{p p} * G^{p p}=\delta(t-r)
\end{aligned}
$$

where ${ }^{*}$ denotes time integration as in Eq. (25) and the damping functions $\eta$ are given by

$$
\begin{aligned}
& \eta_{i j}^{u u}(\mathbf{k}, t, r)=-\int_{\mathbf{k}=\mathbf{p}+\mathbf{q}} d \mathbf{p} d \mathbf{q} \Gamma_{i m n}^{1}(\mathbf{k}, \mathbf{p}, \mathbf{q}) G_{n p}^{u u}(\mathbf{q}, t, r) \Gamma_{p r j}^{1}(\mathbf{q},-\mathbf{p}, \mathbf{k}) Q_{m r}(\mathbf{p}, t, r) \\
& \eta_{i}^{u p}(\mathbf{k}, t, r)=-\int_{\mathbf{k}=\mathbf{p}+\mathbf{q}} d \mathbf{p} d \mathbf{q} \Gamma_{i m n}^{1}(\mathbf{k}, \mathbf{p}, \mathbf{q}) G_{n}^{u p}(\mathbf{q}, t, r) \Gamma_{r}^{2}(\mathbf{q},-\mathbf{p}, \mathbf{k}) Q_{m r}(\mathbf{p}, t, r) \\
& \eta_{j}^{p u}(\mathbf{k}, t, r)=-\int_{\mathbf{k}=\mathbf{p}+\mathbf{q}} d \mathbf{p} d \mathbf{q} \Gamma_{m}^{2}(\mathbf{k}, \mathbf{p}, \mathbf{q}) G_{n}^{p u}(\mathbf{q}, t, r) \Gamma_{n r j}^{1}(\mathbf{q},-\mathbf{p}, \mathbf{k}) Q_{r m}(\mathbf{p}, t, r) \\
& \eta^{p p}(\mathbf{k}, t, r)=-\int_{\mathbf{k}=\mathbf{p}+\mathbf{q}} d \mathbf{p} d \mathbf{q} \Gamma_{m}^{2}(\mathbf{k}, \mathbf{p}, \mathbf{q}) G^{p p}(\mathbf{q}, t, r) \Gamma_{n}^{2}(\mathbf{q},-\mathbf{p}, \mathbf{k}) Q_{m n}(\mathbf{p}, t, r)
\end{aligned}
$$


Now introduce the normalized Helmholtz decomposition of the field $u_{i}$ :

$$
u_{i}(\mathbf{k}, t)=w_{i}(\mathbf{k}, t)+i k_{i} k^{-1} \phi(\mathbf{k}, t)
$$

The isotropy of the lowest order fluctuating fields implies that the damping and response functions must have the form

$$
\begin{aligned}
& \eta_{i j}^{u u}(\mathbf{k}, t, r)=\eta^{w w}(k, t, r) P_{i j}(\mathbf{k})+\eta^{\phi \phi}(k, t, r) P_{i j}^{*}(\mathbf{k}) \\
& \eta_{i}^{u p}(\mathbf{k}, t, r)=\eta^{\phi p}(k, t, r) i k_{i} k^{-1} \\
& \eta_{j}^{p u}(\mathbf{k}, t, r)=-\eta^{p \phi}(k, t, r) i k_{j} k^{-1}
\end{aligned}
$$

and

$$
\begin{aligned}
& G_{i j}^{u u}(\mathbf{k}, t, s)=G^{w w}(k, t, s) P_{i j}(\mathbf{k})+G^{\phi \phi}(k, t, s) P_{i j}^{*}(\mathbf{k}) \\
& G_{i}^{u p}(\mathbf{k}, t, s)=G^{\phi p}(k, t, s) i k_{i} k^{-1} \\
& G_{j}^{p u}(\mathbf{k}, t, s)=-G^{p \phi}(k, t, s) i k_{j} k^{-1}
\end{aligned}
$$

In Eqs. (30) and (31), $P_{i j}$ and $P_{i j}^{*}$ are the transverse and longitudinal projection operators

$$
\begin{aligned}
& P_{i j}(\mathbf{k})=\delta_{i j}-k_{i} k_{j} k^{-2} \\
& P_{i j}^{*}(\mathbf{k})=k_{i} k_{j} k^{-2}
\end{aligned}
$$

Substitute Eqs. (30) and (31) into the governing equations Eq. (28) and separate transverse and longitudinal components. The result is that $G^{w w}$ decouples from the rest of the system and satisfies, in the abridged notation of Eq. (28),

$$
\dot{G}^{w w}+\eta^{w w} * G^{w w}=\delta(t-r)
$$

while the remaining response functions satisfy the system

$$
\begin{aligned}
& \left\{\left[\begin{array}{ll}
1 & 0 \\
0 & 1
\end{array}\right] \frac{\partial}{\partial t}+\left[\begin{array}{cc}
0 & k \rho_{0}^{-1} \\
-\rho_{0} c^{2} k & 0
\end{array}\right]\right\}\left[\begin{array}{cc}
G^{\phi \phi} & G^{\phi p} \\
G^{p \phi} & G^{p p}
\end{array}\right] \\
& +\left[\begin{array}{ll}
\eta^{\phi \phi} & \eta^{\phi p} \\
\eta^{p \phi} & \eta^{p p}
\end{array}\right] *\left[\begin{array}{ll}
G^{\phi \phi} & G^{\phi p} \\
G^{p \phi} & G^{p p}
\end{array}\right]=\delta(t-r)\left[\begin{array}{ll}
1 & 0 \\
0 & 1
\end{array}\right]
\end{aligned}
$$


The solution of Eq. (33) for the response equations is discussed later. For now, the response matrix with the simplified structure of Eq. (24) is used to derive formal expressions for the transport coefficients.

\section{TSDIA analysis of inhomogeneous weakly compressible turbulence}

TSDIA introduces, in addition to the usual Reynolds averaging, a two-scale decomposition

$$
\begin{aligned}
\frac{\partial}{\partial x_{i}} & =\frac{\partial}{\partial x_{i}^{\prime}}+\frac{\partial}{\partial X_{i}} \\
\frac{\partial}{\partial t} & =\frac{\partial}{\partial t^{\prime}}+\frac{\partial}{\partial T}
\end{aligned}
$$

where $\mathbf{x}^{\prime}$ and $t^{\prime}$ describe small turbulent scales of motion and $\mathbf{X}$ and $T$ describe large scale flow features including inhomogeneity and anisotropy. It is natural to assume that the mean quantities depend only on the slow variables $X, T$. Substitution of the scale decomposition Eq. (34) in the mean equations Eqs. (6)-(8) therefore leaves these equations unaltered. Corresponding to Eq. (34), write the compressible field quantities as

$$
\begin{gathered}
s=s\left(\mathbf{x}^{\prime}, \mathbf{X}, t^{\prime}, T\right) \\
u_{i}=u_{i}\left(\mathbf{x}^{\prime}, \mathbf{X}, t^{\prime}, T\right) \\
p=p\left(\mathbf{x}^{\prime}, \mathbf{X}, t^{\prime}, T\right)
\end{gathered}
$$

and apply the scale decompositions of Eq. (34) to the equations for fluctuations Eqs. (18)(20). With these substitutions, dropping primes in the small scale variables, the fluctuation equations take the form

$$
\begin{aligned}
& \frac{\partial s}{\partial t}+\mathbf{U} \cdot \nabla_{x} s+\mathbf{u}^{\infty} \cdot \nabla_{x} s=-\mathcal{F}^{s} \\
& \frac{\partial \mathbf{u}}{\partial t}+\mathbf{U} \cdot \nabla_{x} \mathbf{u}+\mathbf{u}^{\infty} \cdot \nabla_{x} \mathbf{u}+\mathbf{u} \cdot \nabla_{x} \mathbf{u}^{\infty}+\frac{1}{R} \nabla_{x} p=-\mathcal{F}^{u} \\
& \frac{\partial p}{\partial t}+\mathbf{U} \cdot \nabla_{x} p+\mathbf{u}^{\infty} \cdot \nabla_{x} p+R c^{2} \nabla_{x} \cdot \mathbf{u}=-\mathcal{F}^{p}
\end{aligned}
$$

where

$$
\mathcal{F}^{s}=\left(\frac{\partial s}{\partial T}+\mathbf{U} \cdot \nabla_{X} s\right)+\mathbf{u}^{\infty} \cdot \nabla_{X} S+\mathbf{u} \cdot \nabla_{X} S
$$




$$
\begin{aligned}
\mathcal{F}^{u} & =\left(\frac{\partial \mathbf{u}}{\partial T}+\mathbf{U} \cdot \nabla_{X} \mathbf{u}\right)+\mathbf{u}^{\infty} \cdot \nabla_{X} \mathbf{u}+\mathbf{u} \cdot \nabla_{X} \mathbf{u}^{\infty}+\frac{1}{R} \nabla_{X} p \\
& -\frac{\rho}{R^{2}} \nabla_{x} p^{\infty}-\frac{\rho}{R^{2}} \nabla_{X} p^{\infty}+\mathbf{u} \cdot \nabla_{X} \mathbf{U}-\frac{\rho}{R^{2}} \nabla_{X} P-\frac{\rho}{R^{2}} \nabla_{X} p-\frac{\rho}{R^{2}} \nabla_{x} p \\
& +\mathbf{u}^{\infty} \cdot\left(\nabla_{X} U\right)^{C} \\
\mathcal{F}^{p} & =\left(\frac{\partial p}{\partial T}+\mathbf{U} \cdot \nabla_{X} p\right)+\mathbf{u}^{\infty} \cdot \nabla_{X} p+R c^{2} \nabla_{X} \cdot u \\
& +\mathbf{u} \cdot \nabla_{X} p^{\infty}+\mathbf{u} \cdot \nabla_{x} p^{\infty}+\gamma p^{\infty} \nabla_{X} \cdot \mathbf{u}+\gamma p^{\infty} \nabla_{x} \cdot \mathbf{u} \\
& +\left(\frac{\partial p^{\infty}}{\partial T}+\mathbf{U} \cdot \nabla_{X} p^{\infty}\right)+\mathbf{U} \cdot \nabla_{x} p^{\infty}+\mathbf{u}^{\infty} \cdot \nabla_{X} P+\gamma p^{\infty} \nabla_{X} \cdot U \\
& +\gamma p \nabla_{X} \cdot U+\mathbf{u} \cdot \nabla_{X} P+\mathbf{u}^{\infty} \cdot \nabla_{X} p^{\infty}+\left(\frac{\partial p^{\infty}}{\partial t}+\mathbf{u}^{\infty} \cdot \nabla_{x} p^{\infty}\right)
\end{aligned}
$$

In Eqs. (35)-(40), the variable mean density $R$ and sound speed $c$ appear because we now consider weakly inhomogeneous fields. The averages in Eqs. (15)-(17), which maintain the property that the fluctuation equations have zero mean, have been dropped in Eqs. (38)-(40) because they do not contribute to the formation of moments at the level of approximation to be introduced later.

Since the lowest order field is locally homogeneous, a spatial Fourier transform is again appropriate. The spatial part of the decomposition Eq. (34) becomes

$$
\frac{\partial}{\partial x_{i}}=i k_{i}+\frac{\partial}{\partial X_{i}}
$$

With these assumptions, the fluctuating fields are expressed in terms of the corresponding forces and response functions as

$$
\begin{aligned}
s(\mathbf{k}, \mathbf{X}, t, T) & =-\int_{0}^{t} d r G^{s s}(\mathbf{k}, \mathbf{X}, t, r, T) \mathcal{F}^{s}(\mathbf{k}, \mathbf{X}, r, T) \\
u_{i}(\mathbf{k}, \mathbf{X}, t, T) & =-\int_{0}^{t} d r\left[G_{i j}^{u u}(\mathbf{k}, \mathbf{X}, t, r, T) \mathcal{F}_{j}^{u}(\mathbf{k}, \mathbf{X}, r, T)\right. \\
& \left.+G_{i}^{u p}(\mathbf{k}, \mathbf{X}, t, r, T) \mathcal{F}^{p}(\mathbf{k}, \mathbf{X}, r, T)\right] \\
p(\mathbf{k}, \mathbf{X}, t, T) & =-\int_{0}^{t} d r\left[G^{p p}(\mathbf{k}, \mathbf{X}, t, r, T) \mathcal{F}^{p}(\mathbf{k}, \mathbf{X}, r, T)\right. \\
& \left.+G_{j}^{p u}(\mathbf{k}, \mathbf{X}, t, r, T) \mathcal{F}_{j}^{u}(\mathbf{k}, \mathbf{X}, r, T)\right]
\end{aligned}
$$

The possibility of writing explicit expressions like Eqs. (41)-(43) is an important advantage of the DIA formalism. But it must be emphasized that these expressions can only be used to form moments containing $s, u_{i}$, and $p$ : DIA provides statistical formulas for the field quantities, not pointwise solutions of the equations of motion. 
Analytically, local homogeneity means that the various response and correlation functions $G(\mathbf{k}, \mathbf{X}, t, r, T)$ and $Q(\mathbf{k}, \mathbf{X}, t, r, T)$ are evaluated as the corresponding homogeneous quantities $G(\mathbf{k}, t, r)$ and $Q(\mathbf{k}, t, r)$ but with all single point moments which characterize the fields treated as functions of $X, T$. For example, consider the typical forms ${ }^{1}$ for the response and correlation functions of isotropic incompressible turbulence,

$$
\begin{aligned}
G(k, t, r) & =\exp [-\eta(k)(t-r)] H(t-r) \\
Q(k, t, r) & =\frac{1}{4 \pi} C_{K} \varepsilon^{2 / 3} k^{-11 / 3} \exp [-\eta(k)|t-r|] \\
\eta(k) & =C_{D} \varepsilon^{1 / 3} k^{2 / 3} \\
k & \geq k_{0}
\end{aligned}
$$

where $H$ is the unit step function. These expressions are generalized to weakly inhomogeneous incompressible turbulence by letting $\varepsilon$ and $k_{0}$ depend on $X$ and $T$.

With these results, it is straightforward to evaluate the moments needed to close the mean flow equations. In evaluating the moments, the following truncations of the series which the TSDIA generates are adopted:

(a) only modeled terms which are at most linear in the mean gradients are retained,

(b) only modeled terms with at most second order derivatives with respect to $X, T$ are retained,

(c) modeled terms derived by repeated convolution with the compressible response functions are dropped.

Whereas (a) and (b) follow from the formal development of TSDIA as a perturbation expansion, (c) is an additional approximation.

In the following, dependence on $X, T$ will be understood but not written explicitly.

\section{A. Entropy velocity correlation}

The entropy velocity correlation appears in the mean entropy equation Eq. (6). Write this correlation as the integral

$$
<u_{i}^{\infty} s>=\int d \mathbf{k}<u_{i}^{\infty}(-\mathbf{k}, t) s(\mathbf{k}, t)>
$$


Substitute the expression for $s$ in terms of its response function Eq. (41) in Eq. (45) to obtain

$$
\begin{aligned}
-<u_{i}^{\infty} s> & =\int d \mathbf{k} \int_{0}^{t} d r G^{s s}(\mathbf{k}, t, r) \times \\
\{ & <u_{i}^{\infty}(-\mathbf{k}, t) \frac{\partial s}{\partial T}(\mathbf{k}, r)> \\
& +<u_{i}^{\infty}(-\mathbf{k}, t) U_{p} \frac{\partial s}{\partial X_{p}}(\mathbf{k}, r)> \\
& +<u_{i}^{\infty}(-\mathbf{k}, t) \dot{u}_{j}^{\infty}(\mathbf{k}, r)>\frac{\partial S}{\partial X_{j}} \\
& \left.+<u_{i}^{\infty}(-\mathbf{k}, t) u_{j}(\mathbf{k}, r)>\frac{\partial S}{\partial X_{j}}\right\}
\end{aligned}
$$

The formation of nonzero correlations between $\mathbf{u}^{\infty}$ and compressible fields in lines $(46 a),(46 b),(46 d)$ requires an additional time integration over the response functions; the approximation (c) above suppresses these terms. It can be noted that some of these terms will also generate products of gradients and gradients of higher order which are also excluded by (a) and (b).

Term (46c) contributes a gradient transport expression

$$
-<u_{i}^{\infty}(-\mathbf{k}, t) s(\mathbf{k}, t)>=\int d \mathbf{k} \int_{0}^{t} d r G^{s s}(\mathbf{k}, t, r) Q_{i j}(\mathbf{k}, t, r) \frac{\partial S}{\partial X_{j}}
$$

To lowest order of TSDIA, the integral in Eq. (47) is evaluated by substituting isotropic quasi-homogeneous forms for $G^{s s}$ and $Q_{i j}$ :

$$
\begin{aligned}
Q_{i j}(\mathbf{k}, t, r) & =Q(k, t, r) P_{i j}(\mathbf{k}) \\
G^{s \boldsymbol{s}}(\mathbf{k}, t, r) & =G^{s \boldsymbol{s}}(k, t, r)
\end{aligned}
$$

To construct a single point model, it is assumed that the fast time scale dependence is stationary. Therefore, the response and correlation functions depend only the time difference $\tau=t-r$ :

$$
\begin{gathered}
Q(k, t, r)=Q(k, t-r)=Q(k, \tau) \\
G^{s s}(k, t, r)=G^{s s}(k, t-r)=G^{s s}(k, \tau)
\end{gathered}
$$


Furthermore, since the turbulent time scales are much shorter than those of the mean motion, the long time limit $t \rightarrow \infty$ is taken. Substituting Eqs. (48) and (49) in Eq. (47) and taking the long time limit results in the gradient transport model

$$
-<u_{i}^{\infty} s>=\nu^{s s} \frac{\partial S}{\partial X_{i}}
$$

where the transport coefficient is defined by

$$
\nu^{s s}=\frac{2}{3} \int_{0}^{\infty} 4 \pi k^{2} d k \int_{0}^{\infty} d \tau G^{s s}(k, \tau) Q(k, \tau)
$$

The factor of $2 / 3$ arises from angular integration of the transverse projection operator $P_{i j}$. This type of expression for a transport coefficient is familiar from Yoshizawa's TSDIA calculations. ${ }^{1,4}$

\section{B. Velocity pressure correlations}

Four such correlations appear in Eq. (8) for the mean pressure: $\left\langle\mathbf{u}^{\infty} p^{\infty}\right\rangle$, $\left.<\mathbf{u}^{\infty} p\right\rangle,\left\langle\mathbf{u} p^{\infty}>\right.$, and $\left\langle\mathbf{u} \cdot \nabla p^{\infty}\right\rangle$.

1. The correlation $\left\langle\mathbf{u}^{\infty} p^{\infty}\right\rangle$

The incompressible correlation $\left\langle\mathbf{u}^{\infty} p^{\infty}>\right.$ has been analyzed by Shimomura ${ }^{20}$ who finds that it is a diffusion of turbulence quantities. In terms of single point quantities, the integrals become

$$
-<p^{\infty} u_{i}^{\infty}>=\frac{K^{2}}{\varepsilon}\left(C_{1} \frac{\partial K}{\partial X_{i}}+C_{2} \frac{K}{\varepsilon} \frac{\partial \varepsilon}{\partial X_{i}}\right)
$$

Terms of this type will also appear in the closure of correlations containing compressible fields and will be discussed below.

2. The correlation $\left\langle\mathbf{u}^{\infty} p\right\rangle$

Write the second velocity pressure correlation as the integral

$$
\begin{aligned}
-<u_{i}^{\infty} p> & =-\int d \mathbf{k}<u_{i}^{\infty}(-\mathbf{k}, t) p(\mathbf{k}, t)> \\
& =\int d \mathbf{k} \int_{0}^{t} d r\left\{G^{p p}(\mathbf{k}, t, r)<u_{i}^{\infty}(-\mathbf{k}, t) \mathcal{F}^{p}(\mathbf{k}, r)>\right. \\
& \left.+G_{j}^{p u}(\mathbf{k}, t, r)<u_{i}^{\infty}(-\mathbf{k}, t) \mathcal{F}_{j}^{u}(\mathbf{k}, r)>\right\}
\end{aligned}
$$


Closure of this moment requires that the forces from Eqs. (38)-(40) be substituted in Eq. (53). The approximation in which only one time convolution with a compressible response function is allowed shows, as for entropy correlations, that we need only consider terms containing correlations of the incompressible field with itself; the result of evaluating only such terms appears in Appendix II, Sect. A.

Further analysis shows that some of these terms vanish. A list of the nonvanishing terms also appears in Appendix II, Sect. A. Among these terms, some have the form familiar from eddy viscosity modeling: they are (1) proportional to gradients of mean fields, and (2) nonvanishing in regions of constant turbulence properties. Property (2) gives these terms the character of enhancements of molecular properties due to turbulent fluctuations; the molecular properties themselves are the result instead of thermal fluctuations. The transport coefficient $\nu^{s s}$ of Eq. (51) is an example. Such terms will be denoted by the subscript $M$; only these terms will be evaluated in detail in this paper.

But TSDIA also generates a second set of terms which depend on gradients of turbulence quantities. Shimomura's closure of the pressure velocity correlation Eq. (52) is of this type. Unlike the terms just described, these terms vanish when the turbulence properties are constant. Since turbulence properties are rarely constant in practice, it is an important and nontrivial prediction of TSDIA that such corrections to the transport model exist. Because of the large number of such terms and the lengthy analysis required to evaluate many of them, we defer a complete analysis of these terms to later work. In this paper, such terms will be labelled with the subscript $T$. A preliminary description of them appears in Appendix III.

With these conventions,

$$
<\mathbf{u}^{\infty} p>=<\mathbf{u}^{\infty} p>_{M}+<\mathbf{u}^{\infty} p>_{T}
$$

It is shown in Appendix II, Sect. A that

$$
-<u_{i}^{\infty} p>_{M}=\nu_{1}^{p p} \frac{\partial P}{\partial X_{i}}
$$

where, repeating the argument which led to Eq. (51), the transport coefficient $\nu_{1}^{p p}$ is given by

$$
\nu_{1}^{p p}=\frac{2}{3} \int_{0}^{\infty} 4 \pi k^{2} d k \int_{0}^{\infty} d \tau G^{p p}(k, \tau) Q(k, \tau)
$$


3. The correlation $\left\langle\mathbf{u} p^{\infty}\right\rangle$

Write the third velocity pressure correlation as the integral

$$
\begin{aligned}
-<u_{i} p^{\infty}> & =-\int d \mathbf{k}<p^{\infty}(-\mathbf{k}, t) u_{i}(\mathbf{k}, t)> \\
& =\int d \mathbf{k} \int_{0}^{t} d r\left\{G_{i}^{u p}(\mathbf{k}, t, r)<p^{\infty}(-\mathbf{k}, t) \mathcal{F}^{p}(\mathbf{k}, r)>\right. \\
& \left.+G_{i j}^{u u}(\mathbf{k}, t, r)<p^{\infty}(-\mathbf{k}, t) \mathcal{F}_{j}^{u}(\mathbf{k}, r)>\right\}
\end{aligned}
$$

The terms generated by substituting the forces of Eqs. (38)-(40) in Eq. (56) are listed in Appendix II, Sect. B. The result has the form

$$
<\mathbf{u} p^{\infty}>=<\mathbf{u} p^{\infty}>_{M}+<\mathbf{u} p^{\infty}>_{T}
$$

where the mean flow dependent term is

$$
-<u_{i} p^{\infty}>_{M}=-\nu_{2}^{p p} \frac{\partial P}{\partial X_{i}}
$$

and the transport coefficient $\nu_{2}^{p p}$ is given by

$$
\nu_{2}^{p p}=\frac{1}{R^{2} c^{2}} \int_{0}^{\infty} 4 \pi k^{2} d k \int_{0}^{\infty} d \tau\left\{\frac{2}{3} G^{w w}(k, \tau)+\frac{1}{3} G^{\phi \phi}(k, \tau)\right\} Q^{p}(k, \tau)
$$

In Eq. (58), we have assumed, corresponding to Eq. (49) for the two-time velocity correlation function, an isotropic stationary form for the two-time pressure correlation

$$
<p^{\infty}(\mathbf{k}, t) p^{\infty}(\mathbf{k}, r)>=Q^{p}(k, \tau) \delta\left(\mathbf{k}+\mathbf{k}^{\prime}\right)
$$

Whereas the single time pressure correlation function is described by Batchelor's analysis, ${ }^{21}$ calculation of the two-time pressure correlation remains an open problem.

Eq. (57) has an anti-diffusive character, but the complete transport coefficient is $\nu^{p p}=\nu_{1}^{p p}-\nu_{2}^{p p}$ where $\nu_{1}^{p p}$ is evaluated in Eq. (55); it will be shown later that the negative contribution is smaller in magnitude than the positive contribution by a factor of the order of the turbulent Mach number squared. 
4. The correlation $\left\langle\mathbf{u} \cdot \nabla p^{\infty}\right\rangle$

The fourth pressure velocity correlation is written as an integral

$$
\begin{aligned}
& -<\mathbf{u} \cdot \nabla p^{\infty}>=\int d \mathbf{k} \int_{0}^{t} d r \\
& \left\{<G_{i}^{u p}(\mathbf{k}, t, r) \mathcal{F}^{p}(\mathbf{k}, r)\left(-i k_{i}+\frac{\partial}{\partial X_{i}}\right) p^{\infty}(-\mathbf{k}, t)>\right. \\
& \left.+<G_{i j}^{u u}(\mathbf{k}, t, r) \mathcal{F}_{j}^{u}(\mathbf{k}, r)\left(-i k_{j}+\frac{\partial}{\partial X_{j}}\right) p^{\infty}(-\mathbf{k}, t)>\right\}
\end{aligned}
$$

It is shown in Appendix II, Sect. C that the only mean flow dependent term is

$$
-<\mathbf{u} \cdot \nabla_{x} p^{\infty}>_{M}=\Pi \frac{\partial U_{m}}{\partial X_{m}}
$$

where the effective pressure $\Pi$ is given by

$$
\Pi=\gamma \int_{0}^{\infty} 4 \pi k^{2} d k \int_{0}^{\infty} d \tau k G^{\phi p}(k, \tau) Q^{p}(k, \tau)
$$

\section{Velocity-velocity correlations}

The velocity-velocity correlations arise in the mean momentum equation Eq.

There are three such correlations: $\left\langle\mathbf{u}^{\infty} \mathbf{u}^{\infty}\right\rangle,\left\langle\mathbf{u}^{\infty} \mathbf{u}\right\rangle$, and $\left\langle\mathbf{u} \cdot \nabla \mathbf{u}^{\infty}\right\rangle$

1. The correlation $\left\langle\mathbf{u}^{\infty} \mathbf{u}^{\infty}\right\rangle$

The incompressible correlation $\left\langle\mathbf{u}^{\infty} \mathbf{u}^{\infty}>\right.$ has been extensively investigated by Yoshizawa. ${ }^{1}$ At the level of approximation evaluated here, the result of the TSDIA analysis is the usual linear eddy viscosity representation

$$
-<u_{i}^{\infty} u_{j}^{\infty}>=-\frac{2}{3} K \delta_{i j}+\nu\left(S_{i j}-\frac{1}{3} S_{k k} \delta_{i j}\right)
$$

where $S_{i j}$ is the strain rate

$$
S_{i j}=\frac{\partial U_{i}}{\partial X_{j}}+\frac{\partial U_{j}}{\partial X_{j}}
$$

and the incompressible eddy viscosity is the integral ${ }^{1}$

$$
\nu=\frac{4}{15} \int_{0}^{\infty} 4 \pi k^{2} d k \int_{0}^{\infty} d \tau Q(k, \tau) G(k, \tau)
$$

where $G$ is the incompressible velocity response function. 
2. The correlation $\left\langle\mathbf{u}^{\infty} \mathbf{u}\right\rangle$

The second correlation is written as the integral

$$
\begin{aligned}
-<u_{i}^{\infty} u_{j}> & =-\int d \mathbf{k}<u_{i}^{\infty}(-\mathbf{k}, t) u_{j}(\mathbf{k}, t)> \\
& =\int d \mathbf{k} \int_{0}^{t} d r\left\{G_{j m}^{u u}(\mathbf{k}, t, r)<u_{i}^{\infty}(-\mathbf{k}, t) \mathcal{F}_{m}^{u}(\mathbf{k}, r)>\right. \\
& \left.+G_{j}^{u p}(\mathbf{k}, t, r)<u_{i}^{\infty}(-\mathbf{k}, t) \mathcal{F}^{p}(\mathbf{k}, r)>\right\}
\end{aligned}
$$

Reference to Appendix II Sect. D shows that there is only one mean flow dependent term, which can be described as a renormalized bulk viscosity

$$
-<u_{i}^{\infty} u_{j}>_{M}=\nu_{1}^{u u} \frac{\partial U_{m}}{\partial X_{m}} \delta_{i j}
$$

where the transport coefficient is given by

$$
\nu_{1}^{u u}=\frac{1}{3} \int_{0}^{\infty} 4 \pi k^{2} d k \int_{0}^{\infty} d \tau \frac{2}{3} G^{w w}(k, \tau) Q(k, \tau)
$$

3. The correlation $\left\langle\mathbf{u} \cdot \nabla \mathbf{u}^{\infty}\right\rangle$

This correlation is written as the integral

$$
\begin{aligned}
- & <\left(\mathbf{u} \cdot \nabla \mathbf{u}^{\infty}\right)_{i}>=-\int d \mathbf{k}<u_{j}(\mathbf{k}, t)\left(-i k_{j}+\frac{\partial}{\partial X_{j}}\right) u_{i}^{\infty}(-\mathbf{k}, t)> \\
& =\int d \mathbf{k} \int_{0}^{t} d r\left\{G_{j m}^{u u}(\mathbf{k}, t, r)<\mathcal{F}_{m}^{u}(\mathbf{k}, r)\left(-i k_{j}+\frac{\partial}{\partial X_{j}}\right) u_{i}^{\infty}(-\mathbf{k}, t)>\right. \\
& \left.+G_{j}^{u p}(\mathbf{k}, t, r)<\mathcal{F}^{p}(\mathbf{k}, r)\left(-i k_{j}+\frac{\partial}{\partial X_{j}}\right) u_{i}^{\infty}(-\mathbf{k}, t)>\right\}
\end{aligned}
$$

It is shown in Appendix II, Sect. E that the gradient transport term is

$$
-<\left(\mathbf{u} \cdot \nabla \mathbf{u}^{\infty}\right)_{i}>_{M}=\frac{1}{\hat{R}} \frac{\partial P}{\partial X_{i}}
$$

where the coefficient $\hat{R}$ is defined by

$$
\frac{1}{\hat{R}}=\int_{0}^{\infty} 4 \pi k^{2} d k \int_{0}^{\infty} d \tau k G^{\phi p}(k, \tau) Q(k, \tau)
$$

It will be shown later that $\hat{R}$ is related to an effective or renormalized speed of sound waves propagating in the mean flow. This effect has been predicted by Chandrasekhar ${ }^{22}$ and by Staroselski et al. ${ }^{8}$ 


\section{Pressure density correlation}

There are three terms: $\left\langle p^{\infty} p^{\infty}\right\rangle,\left\langle p p^{\infty}\right\rangle$ and $\left\langle s \nabla p^{\infty}\right\rangle$.

1. The correlation $\left\langle p^{\infty} p^{\infty}\right\rangle$

The incompressible pressure correlation $\left\langle p^{\infty} p^{\infty}\right\rangle$ is the subject of Batchelor's classic analysis $^{21}$ which gives

$$
<p^{\infty} p^{\infty}>=C_{P} K^{-2}
$$

where the constant $C_{P}$ can be evaluated theoretically or from measured data. This result also holds in the lowest order of TSDIA.

2. The correlation $\left\langle p p^{\infty}\right\rangle$

For this correlation,

$$
\begin{aligned}
-<p^{\infty} p> & =-\int d \mathbf{k}<p^{\infty}(-\mathbf{k}, t) p(\mathbf{k}, t)> \\
& =\int d \mathbf{k} \int_{0}^{t} d r\left\{G^{p p}(\mathbf{k}, t, r)<p^{\infty}(-\mathbf{k}, t) \mathcal{F}^{p}(\mathbf{k}, r)>\right. \\
& \left.+G_{j}^{p u}(\mathbf{k}, t, r)<p^{\infty}(-\mathbf{k}, t) \mathcal{F}_{j}^{u}(\mathbf{k}, r)>\right\}
\end{aligned}
$$

In Appendix II Sect. F, it is shown that the mean flow dependent term is

$$
-<p p^{\infty}>_{M}=R^{2} c^{2} \nu_{2}^{u u} \frac{\partial U_{p}}{\partial X_{p}}
$$

where the effective bulk viscosity is defined by

$$
R^{2} c^{2} \nu_{2}^{u u}=\gamma \int 4 \pi k^{2} d k \int_{0}^{\infty} d \tau G^{p p}(k, \tau) Q^{p}(k, \tau)
$$

Note finally that since there is no pressure source in $\mathcal{F}^{s}$, the correlation $\left\langle s p^{\infty}\right\rangle$ vanishes to this order.

Introducing only the mean flow dependent terms, Eqs. (50), (54), (57), (59), (61), (63), (65), and (68) computed by TSDIA into Eqs. (6)-(8), we obtain the gradient transport model for weakly compressible turbulence,

$$
\begin{aligned}
& \frac{\partial S}{\partial t}+\mathbf{U} \cdot \nabla S=\nabla \cdot\left(\nu^{s s} \nabla S\right) \\
& \frac{\partial \mathbf{U}}{\partial t}+\mathbf{U} \cdot \nabla \mathbf{U}+\left(\frac{1}{R}-\frac{1}{\hat{R}}\right) \nabla P=\nabla \cdot\left\{-\frac{2}{3} K \mathbf{I}+\nu\left[\nabla \mathbf{U}+(\nabla \mathbf{U})^{T}\right]^{S}\right\}+\nabla\left(\nu^{u u} \nabla \cdot \mathbf{U}\right) \\
& \frac{\partial P}{\partial t}+\mathbf{U} \cdot \nabla P+(\gamma-\hat{\gamma}) P \nabla \cdot \mathbf{U}=\nabla \cdot\left(\nu^{p p} \nabla P\right)
\end{aligned}
$$


The gradients of turbulence quantities generated by TSDIA can of course be added as well. A preliminary qualitative description of these terms and a discussion of the form of the resulting model appears in Appendix III.

The transport coefficients in Eqs. (70)-(72) are found from Eqs. (51), (55), (58), (60), $(62),(64),(66)$, and (69) above and are summarized here for convenient reference as

$$
\begin{aligned}
\nu & =\frac{4}{15} \int_{0}^{\infty} 4 \pi k^{2} d k \int_{0}^{\infty} d \tau G(k, \tau) Q(k, \tau) \\
\nu^{s s} & =\frac{2}{3} \int_{0}^{\infty} 4 \pi k^{2} d k \int_{0}^{\infty} d \tau G^{s s}(k, \tau) Q(k, \tau) \\
\nu^{p p} & =\frac{2}{3} \int_{0}^{\infty} 4 \pi k^{2} d k \int_{0}^{\infty} d \tau G^{p p}(k, \tau) Q(k, \tau) \\
& -\frac{1}{c^{2}} \int_{0}^{\infty} 4 \pi k^{2} d k \int_{0}^{\infty} d \tau\left\{\frac{2}{3} G^{w w}(k, \tau)+\frac{1}{3} G^{\phi \phi}(k, \tau)\right\} Q^{p}(k, \tau) \\
\hat{\gamma} & =(\gamma-1) \Pi / P \\
\Pi & =\gamma \int_{0}^{\infty} 4 \pi k^{2} d k \int_{0}^{\infty} d \tau k G^{\phi p}(k, \tau) Q^{p}(k, \tau) \\
\nu^{u u} & =\frac{1}{3} \int_{0}^{\infty} 4 \pi k^{2} d k \int_{0}^{\infty} d \tau \frac{2}{3} G^{w w}(k, \tau) Q(k, \tau) \\
& -\frac{\gamma}{R^{2} c^{2}} \int_{0}^{\infty} 4 \pi k^{2} d k \int_{0}^{\infty} d \tau G^{p p}(k, \tau) Q^{p}(k, \tau) \\
\frac{1}{\hat{R}} & =\int_{0}^{\infty} 4 \pi k^{2} d k \int_{0}^{\infty} d \tau k G^{\phi p}(k, \tau) Q(k, \tau)
\end{aligned}
$$

The eddy viscosity $\nu$ and eddy diffusivity $\nu^{s s}$ are transport terms of the type familiar from studies of incompressible turbulence. The term $\nu^{u u}$ is an effective bulk viscosity generated by the compressible fluctuations. The modified pressure $\Pi$ has been introduced into the transport model Eq. (71) through the modified specific heat ratio $\hat{\gamma}$ defined by Eq. (76). The turbulent fluctuations therefore modify not only the viscosities seen by the mean flow, but also the effective $\gamma$. Of course, the thermodynamic specific heat ratio is unchanged. The TSDIA expressions for $\Pi$ demonstrate that this modification of $\gamma$ is a long time effect: at short times, $\Pi \sim t^{2}$, hence $\hat{\gamma} \sim \gamma+O\left(t^{2}\right)$.

To clarify the role of the $\hat{R}$ term, note that if the mean field is treated as an ensemble mean, then it can contain non-random sound waves. The propagation speed of these waves can be found by linearizing the mean flow equations about a constant state, setting

$$
P=P_{0}+P^{\prime}
$$




$$
\begin{aligned}
& \mathbf{U}=\mathbf{U}_{0}+\mathbf{U}^{\prime} \\
& R=R_{0}+R^{\prime}
\end{aligned}
$$

where $P_{0}$ and $R_{0}$ satisfy the thermodynamic relation $c_{0}=\left(\gamma P_{0} / R_{0}\right)^{1 / 2}$ where $c_{0}$ is the sound speed at constant pressure $P_{0}$ and density $R_{0}$. The standard derivation shows that sound waves propagate in the mean field with speed

$$
C^{2}=\left[\gamma P_{0}-(\gamma-1) \Pi\right]\left[\frac{1}{R_{0}}-\frac{1}{\hat{R}}\right]=c_{0}^{2}\left[1-\gamma(\gamma-1) \frac{\Pi}{R_{0} c^{2}}\right]\left[1-\frac{R_{0}}{\hat{R}}\right]
$$

By evaluating the terms $\Pi$ and $\hat{R}$ explicitly later, it will be shown that Eq. (80) corresponds to an increase in the effective speed of sound in the mean flow. An argument for the enhancement of the speed of sound by compressible fluctuations is given by Chandrasekhar; ${ }^{22}$ Staroselski et $\mathrm{al}^{8}$ discuss this effect in the case of strong compressibility.

\section{Evaluation of the transport coefficients}

Explicit expressions for the response functions are needed in order to evaluate Eqs. (73)-(79) for the transport coefficients. In TSDIA calculations ${ }^{1}$ for incompressible turbulence, the required expressions are given the Kolmogorov inertial range forms Eq. (44). In the present case, it is necessary to solve Eqs. (28) for the compressible response functions. The solution of these equations is difficult because the damping factors $\eta$ of Eq. (29) depend on the response functions themselves. Although these equations are already the result of several approximations, the desirability of analytical expressions for the transport coefficients justifies further approximations.

First, it will be convenient to replace the damping by Markovian damping by setting

$$
\eta(k, t, r)=\eta(k) \delta(t-r)
$$

For time stationarity, appropriate to the lowest order TSDIA field,

$$
\eta(k)=\int_{0}^{\infty} d \tau \eta(k, \tau)
$$

where $\tau=t-r$ denotes time difference. Equivalently, in frequency space

$$
\eta(\hat{k})=\eta(k, 0)=\eta(k)
$$


This Markovianization is familiar in statistical physics ${ }^{23}$ and is implicit in all turbulence modeling. It essentially states that turbulent processes occur much faster than mean flow processes. When this assumption is not valid, universal single time turbulence modeling is impossible in principle. Further discussion can be found in the recent work of Yoshizawa ${ }^{24}$ and Woodruff. ${ }^{25}$ We note that the fluctuation-dissipation relation which connects the twotime correlation and the response function in Eq. (44) is justified for Markovianized theories. $^{25}$

The time stationary form of the response equations Eq. (33) which includes the initial conditions is

$$
\left\{\left[\begin{array}{cc}
-i \omega & k / \rho_{0} \\
-\rho_{0} c^{2} k & -i \omega
\end{array}\right]+\left[\begin{array}{cc}
\eta^{\phi \phi} & \eta^{\phi p} \\
\eta^{p \phi} & \eta^{p p}
\end{array}\right]\right\}\left[\begin{array}{cc}
G^{\phi \phi} & G^{\phi p} \\
G^{p \phi} & G^{p p}
\end{array}\right]=\left[\begin{array}{cc}
1 & 0 \\
0 & 1
\end{array}\right]
$$

The Markovianized damping factors Eq. (29) with Eq. (81) can be rewritten as

$$
\begin{aligned}
& \eta^{w w}(\mathbf{k})=-\frac{1}{2} P_{i j}(\mathbf{k}) \int_{0}^{\infty} d \tau \int_{\mathbf{k}=\mathbf{p}+\mathbf{q}} d \mathbf{p} d \mathbf{q} \Gamma_{i m n}^{1}(\mathbf{k}, \mathbf{p}, \mathbf{q}) G^{w w}(\mathbf{q}, \tau) \times \\
& P_{n p}(\mathbf{q}) \Gamma_{p r j}^{1}(\mathbf{q},-\mathbf{p}, \mathbf{k}) Q_{m r}(\mathbf{p}, \tau) \\
&-\frac{1}{2} P_{i j}(\mathbf{k}) \int_{0}^{\infty} d \tau \int_{\mathbf{k}=\mathbf{p}+\mathbf{q}} d \mathbf{p} d \mathbf{q} \Gamma_{i m n}^{1}(\mathbf{k}, \mathbf{p}, \mathbf{q}) G^{\phi \phi}(\mathbf{q}, \tau) \times \\
& P_{n p}^{*}(\mathbf{q}) \Gamma_{p r j}^{1}(\mathbf{q},-\mathbf{p}, \mathbf{k}) Q_{m r}(\mathbf{p}, \tau) \\
& \eta^{\phi \phi}(\mathbf{k})=-P_{i j}^{*}(\mathbf{k}) \int_{0}^{\infty} d \tau \int_{\mathbf{k}=\mathbf{p}+\mathbf{q}} d \mathbf{p} d \mathbf{q} \Gamma_{i m n}^{1}(\mathbf{k}, \mathbf{p}, \mathbf{q}) G^{w w}(\mathbf{q}, \tau) \times \\
& P_{n p}(\mathbf{q}) \Gamma_{p r j}^{1}(\mathbf{q},-\mathbf{p}, \mathbf{k}) Q_{m r}(\mathbf{p}, \tau) \\
&-P_{i j}^{*}(\mathbf{k}) \int_{0}^{\infty} d \tau \int_{\mathbf{k}=\mathbf{p}+\mathbf{q}} d \mathbf{p} d \mathbf{q} \Gamma_{i m n}^{1}(\mathbf{k}, \mathbf{p}, \mathbf{q}) G^{\phi \phi}(\mathbf{q}, \tau) \times \\
& P_{n p}^{*}(\mathbf{q}) \Gamma_{p r j}^{1}(\mathbf{q},-\mathbf{p}, \mathbf{k}) Q_{m r}(\mathbf{p}, \tau) \\
& \eta^{\phi p}(\mathbf{k})=i k_{i} k^{-1} \int_{0}^{\infty} d \tau \int_{\mathbf{k}=\mathbf{p}+\mathbf{q}} d \mathbf{p} d \mathbf{q} \Gamma_{i m n}^{1}(\mathbf{k}, \mathbf{p}, \mathbf{q}) G_{n}^{u p}(\mathbf{q}, \tau) \Gamma_{r}^{2}(\mathbf{q},-\mathbf{p}, \mathbf{k}) Q_{m r}(\mathbf{p}, \tau) \\
& \eta^{p \phi}(\mathbf{k}, \tau)=-i k_{j} k^{-1} \int_{0}^{\infty} d \tau \int_{\mathbf{k}=\mathbf{p}+\mathbf{q}} d \mathbf{p} d \mathbf{q} \Gamma_{m}^{2}(\mathbf{k}, \mathbf{p}, \mathbf{q}) G_{n}^{p u}(\mathbf{q}, \tau) \Gamma_{n r j}^{1}(\mathbf{q},-\mathbf{p}, \mathbf{k}) Q_{r m}(\mathbf{p}, \tau) \\
& \eta^{p p}(\mathbf{k}, \tau)=-\int_{\mathbf{k}=\mathbf{p}+\mathbf{q}} d \mathbf{p} d \mathbf{q} \Gamma_{m}^{2}(\mathbf{k}, \mathbf{p}, \mathbf{q}) G^{p p}(\mathbf{q}, \tau) \Gamma_{n}^{2}(\mathbf{q},-\mathbf{p}, \mathbf{k}) Q_{m n}(\mathbf{p}, \tau)
\end{aligned}
$$

The Markovianized system Eq. (82) for the response functions is solved approximately by perturbing about purely incompressible damping. Note first that Eq. (32) for $G^{w w}$ 
reduces to the equation for the response function of a passive vector field if time integrals containing $G^{\phi \phi}$ are ignored in comparison to time integrals containing $G^{w w}$ in the integral for $\eta^{w w}$ in Eq. (83). The self-consistency of this approximation will be demonstrated later: it will be shown that a consequence of this approximation is that time integrals in $G^{\phi \phi}$ are of order $c^{-2}$ times time integrals in $G^{w w}$. So approximate $G^{w w}$ by the response function of a passive vector field

$$
G^{w w}(k, t, s)=\exp \left[-\eta^{w w}(k)(t-s)\right] H(t-s)
$$

where the damping factor $\eta^{w w}$ is related to the incompressible damping function $\eta$ through

$$
\begin{aligned}
\eta^{w w}(k) & =\alpha_{w} \eta(k) \\
\eta(k) & =C_{D} \varepsilon^{1 / 3} k^{2 / 3}
\end{aligned}
$$

In Eq. (85), $\alpha_{w}$ is the inverse Prandtl number for convection of $w$. This quantity can be evaluated theoretically following the calculation of the Prandtl number for passive fields. ${ }^{26,27}$ Eqs. (83) show that the integrals for the damping factors $\eta^{\phi p}, \eta^{p \phi}, \eta^{p p}$ all contain the corresponding response functions $G^{\phi p}, G^{p \phi}, G^{p p}$. Only the integral for $\eta^{\phi \phi}$ has a contribution which depends on $G^{w w}$. Assume further that time integrals which contain the response functions $G^{\phi p}, G^{p \phi}, G^{p p}$ are small compared to time integrals which contain $G^{w w}$. Then set as a first approximation

$$
\begin{aligned}
\eta^{\phi \phi}(k) & =\alpha_{\phi} \eta(k) \\
\eta^{\phi p}(k) & =\eta^{p \phi}(k)=\eta^{p p}(k)=0
\end{aligned}
$$

where $\alpha_{\phi}$ is another inverse Prandtl number which can be computed by evaluating the integrals in Eq. (83). Like Eq. (84), it will be shown subsequently that this approximation is self consistent: by computing response functions and corrected damping factors, we will find that $\eta^{\phi p}, \eta^{p \phi}, \eta^{p p}$ are of the order $c^{-1}$ times $\eta^{\phi \phi}$. With this initial approximation, the solution of $\mathrm{Eq} .(82)$ is

$$
\begin{aligned}
G^{\phi p}(\hat{k}) & =\frac{-\rho_{0}^{-1} k}{-\omega^{2}+c^{2} k^{2}-i \omega \alpha_{\phi} \eta(k)} \\
G^{p p}(\hat{k}) & =\frac{-i \omega+\alpha_{\phi} \eta(k)}{-\omega^{2}+c^{2} k^{2}-i \omega \alpha_{\phi} \eta(k)} \\
G^{\phi \phi}(\hat{k}) & =\frac{-i \omega}{-\omega^{2}+c^{2} k^{2}-i \omega \alpha_{\phi} \eta(k)} \\
G^{p \phi}(\hat{k}) & =\frac{\rho_{0} c^{2} k}{-\omega^{2}+c^{2} k^{2}-i \omega \alpha_{\phi} \eta(k)}
\end{aligned}
$$


The corresponding time domain expressions for the response functions are

$$
\begin{aligned}
G^{\phi p}(k, \tau) & =-\frac{k}{\rho_{0} S} \exp \left(-\alpha_{\phi} \eta(k) \tau / 2\right) \sin (S \tau) H(\tau) \\
G^{p \phi}(k, \tau) & =\rho_{0} \frac{c^{2} k}{S} \exp \left(-\alpha_{\phi} \eta(k) \tau / 2\right) \sin (S \tau) H(\tau) \\
G^{p p}(k, \tau) & =\exp \left(-\alpha_{\phi} \eta(k) \tau / 2\right) \cos (S \tau) H(\tau) \\
& +\frac{\alpha_{\phi} \eta(k)}{2 S} \exp \left(-\alpha_{\phi} \eta(k) \tau / 2\right) \sin (S \tau) H(\tau) \\
G^{\phi \phi}(k, \tau) & =\exp \left(-\alpha_{\phi} \eta(k) \tau / 2\right) \cos (S \tau) H(\tau) \\
& -\frac{\alpha_{\phi} \eta(k)}{2 S} \exp \left(-\alpha_{\phi} \eta(k) \tau / 2\right) \sin (S \tau) H(\tau)
\end{aligned}
$$

where

$$
S=\left\{c^{2} k^{2}-\frac{1}{4} \alpha_{\phi}^{2} \eta(k)^{2}\right\}^{1 / 2}
$$

This approximation can be improved by substituting the response functions of Eq. (87) into the formulas Eq. (83) for the damping factors. The corrected values of $\eta^{\phi p}, \eta^{p \phi}, \eta^{p p}$ contain terms which oscillate rapidly with frequency $c k$; time integrals of these quantities will consequently be of order $c^{-1}$. Consequently, the corrected $\eta^{\phi p}, \eta^{p \phi}, \eta^{p p}$ are of the order $c^{-1}$ times $\eta^{\phi \phi}$. By solving Eq. (82) for the response functions with this second approximation for the $\eta$ 's, a second approximation to the response matrix is generated. This iteration generates a sequence of approximate solutions to Eq. (82) containing increasingly high order powers of the time scale ratio $\eta(k) / c k$. The approximation Eqs. $(86)-(87)$ is the approximation of lowest order. This approximation has also been stated by Bertoglio et al. ${ }^{6}$

With these approximations, it is possible to evaluate both the transport coefficients of Eqs. (73)-(79) and the correlations of compressible field quantities which enter the two-equation model for weakly compressible turbulence. These correlations, such as the pressure-dilatation and dilatational dissipation, are believed ${ }^{15}$ to be crucial to explaining compressibility effects on mixing layer growth. To evaluate them, it is necessary to solve Eqs. (18)-(20), with all mean field contributions dropped, to express the compressible fields as integrals of the response functions of Eq. (86) and the incompressible source term $p^{\dagger}$ of Eq. (14). The result will express these compressible correlations in terms of the two-time correlation

$$
Q_{i j}^{\dagger}(\mathbf{k}, t, r) \delta\left(\mathbf{k}+\mathbf{k}^{\prime}\right)=<p^{\dagger}(\mathbf{k}, t) p^{\dagger}\left(\mathbf{k}^{\prime}, r\right)>
$$


Although this program is completely routine, $Q^{\dagger}$ is a sixth order correlation of velocity components, therefore explicit evaluation of the required correlations will be quite lengthy. This part of the model development is left to subsequent work.

To evaluate the transport coefficients, we apply the inertial range formula Eq. (44) with the single time correlation function given by the cutoff Kolmogorov spectrum

$$
E(k)=Q(k) / 4 \pi k^{2}= \begin{cases}C_{K^{-}} \varepsilon^{2 / 3} k^{-5 / 3} & \text { if } k_{0} \leq k \leq k_{d} \\ 0 & \text { if } k \leq k_{0} \text { or } k \geq k_{d}\end{cases}
$$

In Eq. (89), $k_{d}$ is proportional to the Kolmogorov scale; the convergence of all integrals in this theory as $k_{d} \rightarrow \infty$ implies that we can set $k_{d}=\infty$ and ignore its finite value. The large scale cutoff $k_{0}$ is defined in terms of the kinetic energy of turbulence $K$ through

$$
K=\frac{3}{2} C_{K} \cdot \varepsilon^{2 / 3} k_{0}^{-2 / 3}
$$

We take also

$$
G^{s s}(k, \tau)=\exp [-\alpha \eta(k) \tau] H(\tau)
$$

where $\alpha$ is the inverse Prandtl number for a passive scalar ${ }^{27}$ Pending a more complete investigation, we postulate for the two time pressure correlation

$$
Q^{p}(k, \tau)=\frac{1}{4 \pi k^{2}} E^{p}(k) \exp \left[-\alpha_{P} \eta(k) \mid \tau \|\right]
$$

where the single time pressure spectrum is given by Batchelor's well-known relation

$$
E^{p}(k)=C_{B} \rho_{0}^{2} \varepsilon^{4 / 3} k^{-7 / 3}
$$

The spectrum constant $C_{B}$ and Prandtl number $1 / \alpha_{P}$ are evaluated theoretically or from data. In evaluating integrals dependent on the term $S$ of Eq. (88), we will make the leading order approximation

$$
S \sim c k
$$

which is consistent with the assumption of small turbulent Mach number. Given these approximations, it is easy to evaluate the transport coefficients in terms of single point quantities. 
The coefficient $\nu^{s s}$ of Eq. (74) is evaluated as

$$
\begin{aligned}
\nu^{s s} & =\frac{2}{3} \int_{0}^{\infty} d k \int_{0}^{\infty} d \tau G^{s s}(k, \tau) E(k) \exp (-\eta \tau) \\
& =\frac{2}{3} \frac{1}{\alpha+1} \int_{0}^{\infty} d k \frac{E(k)}{\eta(k)} \\
& =\frac{2}{3} \frac{1}{\alpha+1} \frac{C_{K}}{C_{D}} \varepsilon^{1 / 3} k_{0}^{-4 / 3} \\
& =\frac{2}{9} \frac{1}{\alpha+1}\left(C_{K} C_{D}\right)^{-1} \frac{K^{-2}}{\varepsilon}
\end{aligned}
$$

The transport coefficient $\nu^{s s}$ describes the convection of a passive scalar, and is therefore of order $M_{t}^{0}$ where the turbulent Mach number is $M_{t}=K^{1 / 2} / c$.

The coefficient $\nu^{p p}$ is evaluated from Eq. (73) as

$$
\begin{aligned}
\nu^{p p} & =\frac{2}{3} \int_{0}^{\infty} d k \int_{0}^{\infty} d \tau G^{p p}(k, \tau) E(k) \exp (-\eta \tau) \\
& -\frac{1}{c^{2}} \int_{0}^{\infty} d k \int_{0}^{\infty} d \tau\left\{\frac{2}{3} G^{w w}(k, \tau)+\frac{1}{3} G^{\phi \phi}(k, \tau)\right\} E^{p}(k) \exp \left(-\alpha_{P} \eta \tau\right) \\
& =\int_{0}^{\infty} d k \frac{2}{3}\left(\alpha_{\phi}+1\right) \frac{\eta}{c^{2} k^{2}} E(k)-\frac{1}{c^{2}} \frac{2}{3} \frac{1}{\alpha_{P}+\alpha_{w}} \frac{E^{p}(k)}{\eta(k)} \\
& =\left[\frac{1}{3}\left(\alpha_{\phi}+1\right) C_{K} C_{D}-\frac{1}{3} \frac{1}{\alpha_{P}+\alpha_{w}} \frac{C_{B}}{C_{D}}\right] \frac{\varepsilon}{c^{2}} k_{0}^{-2} \\
& =\frac{8}{81} C_{K}^{-3}\left[\left(\alpha_{\phi}+1\right) C_{K} C_{D}-\frac{1}{\alpha_{P}+\alpha_{w}} \frac{C_{B}}{C_{D}}\right] M_{t}^{2} \frac{K^{-2}}{\varepsilon}
\end{aligned}
$$

This evaluation is correct to lowest order in $M_{t}$; including the contribution from $G^{\phi \phi}$ would, in view of Eq. (86), lead to corrections of order $M_{t}^{4}$. Diffusion of pressure is therefore an $O\left(M_{t}^{2}\right)$ phenomenon.

In evaluating $\Pi$ from Eq. ( 77 ), we will again compute to leading order in $M_{t}$. Thus,

$$
\begin{aligned}
\Pi & =\gamma \int_{0}^{\infty} 4 \pi k^{3} d k \int_{0}^{\infty} d \tau G^{\phi p}(k, \tau) Q^{p}(k, \tau) \\
& =-\gamma \int_{0}^{\infty} d k \frac{C_{B}}{2 \rho_{0} c^{2}} \varepsilon^{4 / 3} k^{-7 / 3}+O\left(M_{t}^{4}\right) \\
& =-\gamma \frac{<\left(p^{\infty}\right)^{2}>}{2 \rho_{0} c^{2}}
\end{aligned}
$$

where Eq. (67) can be substituted for the total pressure variance so that

$$
\Pi \sim-K M_{t}^{2}
$$


For the bulk viscosity $\nu^{u}$, Eq. (77) implies

$$
\begin{aligned}
\nu^{u u} & =\frac{1}{3} \int_{0}^{\infty} 4 \pi k^{2} d k \int_{0}^{\infty} d \tau \frac{2}{3} G^{w w}(k, \tau) Q(k, \tau) \\
& -\gamma \int 4 \pi k^{2} d k \int_{0}^{\infty} d \tau G^{p p}(k, \tau) Q^{p}(k, \tau)
\end{aligned}
$$

Note that the negative contribution to Eq. (97) is of order $c^{-2}$ compared to the first, therefore it suffices to evaluate the first term only. The result is

$$
\begin{aligned}
\nu^{u u} & =\int_{0}^{\infty} d k \frac{2}{9} \frac{1}{\alpha_{w}+1} \frac{E(k)}{\eta(k)} \\
& =\frac{1}{6} \frac{1}{\alpha_{w}+1} \frac{C_{K}}{C_{D}} \varepsilon^{1 / 3} k_{0}^{-4 / 3} \\
& =\frac{2}{27} \frac{1}{\alpha_{w}+1}\left(C_{K} C_{D}\right)^{-1} \frac{K^{2}}{\varepsilon}
\end{aligned}
$$

Note the somewhat surprising conclusion that the bulk viscosity has an $O\left(M_{t}^{0}\right)$ contribution from the fluctuating $w$ field. The reason is that the decorrelation time of the $w$ field appears in the expressions for the transport coefficients, not its amplitude, and that in the present approximation for the response functions, $w$ decorrelates like a passive vector. Although the bulk viscosity does not vanish in the limit $M_{t} \rightarrow 0$, incompressibility is maintained through the condition that $\nabla \cdot \mathbf{U} \rightarrow 0$.

Evaluating $\hat{R}$ to leading order in $M_{t}$ from Eq. (79),

$$
\begin{aligned}
\frac{1}{\hat{R}} & =\int_{0}^{\infty} 4 \pi k^{2} d k \int_{0}^{\infty} d \tau k G^{\phi p}(k, \tau) Q(k, \tau) \\
& =-\frac{1}{R} \int_{0}^{\infty} d k c^{-2} E(k) \\
& =-\frac{1}{R} M_{t}^{2}
\end{aligned}
$$

Combining the results of Eqs. (96) and (99) in the formula Eq. (80) for the effective speed of mean flow sound waves,

$$
C^{2}=c^{2}\left[1+M_{t}^{2}\right]
$$

since $\Pi / c^{2} \sim M_{t}^{4}$ according to Eq. (96). This result agrees up to the constant multiplying $M_{t}^{2}$ with the prediction of Chandrasekhar ${ }^{22}$ for the speed of propagation of density correlations. 
To summarize, by substituting the leading order terms in $M_{t}$ from Eqs. (93)-(99) for the transport coefficients in Eqs. (70)-(72), we obtain the mean flow equations

$$
\begin{aligned}
& \frac{\partial S}{\partial t}+\mathrm{U} \cdot \nabla S=\nabla \cdot\left(C_{s} \frac{K^{2}}{\varepsilon} \nabla S\right) \\
& \frac{\partial \mathbf{U}}{\partial t}+\mathbf{U} \cdot \nabla \mathbf{U}+\frac{1}{R}\left[1+M_{t}^{2}\right] \nabla P=\nabla \cdot\left\{-\frac{2}{3} \Pi \mathbf{I}+C_{\nu} \frac{K^{2}}{\varepsilon}\left[\nabla \mathbf{U}+(\nabla \mathbf{U})^{T}\right]^{S}\right\} \\
& +\nabla\left(C_{u} \frac{\Pi^{-2}}{\varepsilon} \nabla \cdot \mathrm{U}\right) \\
& \frac{\partial P}{\partial t}+\mathbf{U} \cdot \nabla P+\gamma\left[1+C_{\gamma} M_{t}^{4}\right] P \nabla \cdot \mathbf{U}=\nabla \cdot\left(C_{p} M_{t}^{2} \frac{K^{2}}{\varepsilon} \nabla P\right)
\end{aligned}
$$

where the model constants $C_{s}, C_{\gamma}, C_{p}, C_{\nu}, C_{u}$ are given by explicit integral expressions containing the velocity inertial range constants $C_{K}$ and $C_{D}$ and the incompressible pressure inertial range constants $C_{B}$ and $\alpha_{P}$.

\section{Improved approximate system of equations}

Eqs. (21)-(23) were derived by treating all incompressible pressure terms as perturbations. This approximation lead to the decoupling of the entropy equation and consequently to the simple partitioned structure Eq. (24) of the response matrix. If instead all of the pressure terms in Eqs. (18)-(20) were treated non-perturbatively, the appearance of entropy in the momentum equation would cause the response matrix computed by DIA to be a completely filled three by three block matrix. Even an approximate solution of the corresponding response equations would be more difficult.

However, the decoupling of entropy and the convenient matrix structure of Eq. (24) can be retained by treating only the term coupling $s$ and $p^{\infty}$ as a perturbation. This approximation is particularly reasonable since it is found in numerical simulations ${ }^{28}$ that homogeneous compressible turbulence is nearly isentropic. With this approximation, the response equations still have the form of Eq. (28), but the damping factors become

$$
\begin{aligned}
& \eta_{i j}^{u u}(\mathbf{k}, t, r)=-\int_{\mathbf{k}=\mathbf{p}+\mathbf{q}} d \mathbf{p} d \mathbf{q} \Gamma_{i m n}^{1}(\mathbf{k}, \mathbf{p}, \mathbf{q}) G_{n p}^{u u}(\mathbf{q}, t, r) \Gamma_{p r j}^{1}(\mathbf{q},-\mathbf{p}, \mathbf{k}) Q_{m r}(\mathbf{p}, t, r) \\
& +\frac{\gamma}{c^{2} R^{2}} \int_{\mathbf{k}=\mathbf{p}+\mathbf{q}} d \mathbf{p} d \mathbf{q} p_{i} k_{j} Q^{p}(\mathbf{p}, t, r) G^{p p}(\mathbf{q}, t, r) \\
& \eta_{i}^{u p}(\mathbf{k}, t, r)=-\int_{\mathbf{k}=\mathbf{p}+\mathbf{q}} d \mathbf{p} d \mathbf{q} \Gamma_{i m n}^{1}(\mathbf{k}, \mathbf{p}, \mathbf{q}) G_{n}^{u p}(\mathbf{q}, t, r) \Gamma_{p}^{2}(\mathbf{q},-\mathbf{p}, \mathbf{k}) Q_{m p}(\mathbf{p}, t, r)
\end{aligned}
$$




$$
\begin{aligned}
& +\frac{1}{c^{2} R^{2}} \int_{\mathbf{k}=\mathbf{p}+\mathbf{q}} d \mathbf{p} d \mathbf{q} p_{i} p_{j} G_{j}^{p u}(\mathbf{q}, t, r) Q^{p}(\mathbf{p}, t, r) \\
& \eta_{j}^{p u}(\mathbf{k}, t, r)=-\int_{\mathbf{k}=\mathbf{p}+\mathbf{q}} d \mathbf{p} d \mathbf{q} \Gamma_{p}^{2}(\mathbf{k}, \mathbf{p}, \mathbf{q}) G_{n}^{p u}(\mathbf{q}, t, r) \Gamma_{n r j}^{1}(\mathbf{q},-\mathbf{p}, \mathbf{k}) Q_{r p}(\mathbf{p}, t, r) \\
& -\gamma^{2} \int_{\mathbf{k}=\mathbf{p}+\mathbf{q}} d \mathbf{p} d \mathbf{q} q_{i} k_{j} G_{i}^{u p}(\mathbf{q}, t, r) Q^{p}(\mathbf{p}, t, r) \\
& \eta^{p p}(\mathbf{k}, t, r)=-\int_{\mathbf{k}=\mathbf{p}+\mathbf{q}} d \mathbf{p} d \mathbf{q} \Gamma_{p}^{2}(\mathbf{k}, \mathbf{p}, \mathbf{q}) G^{p p}(\mathbf{q}, t, r) \Gamma_{q}^{2}(\mathbf{q},-\mathbf{p}, \mathbf{k}) Q_{p q}(\mathbf{p}, t, r) \\
& -\frac{\gamma}{c^{2} R^{2}} \int_{\mathbf{k}=\mathbf{p}+\mathbf{q}} d \mathbf{p} d \mathbf{q} q_{i} p_{j} G_{i j}^{u u}(\mathbf{q}, t, r) Q^{p}(\mathbf{p}, t, r)
\end{aligned}
$$

In this approximation, the incompressible pressure correlations appear in the expressions for damping of the compressible fields which take the form

$$
G(k, \tau) \sim \exp \left[-\eta(k) \tau+O\left(M_{t}^{2}\right)\right]
$$

The appearance of $M_{t}$ in the response and time correlation functions is regularizing in the sense that time integrals

$$
\int_{0}^{\infty} d \tau G(k, \tau) Q(k, \tau) \sim \frac{1}{a+O\left(M_{t}^{2}\right)}
$$

and the theory is therefore well behaved even if $M_{t}$ becomes large. This provides a more fundamental view of the role of pressure fluctuations and is suggestive for generalization of this theory to strong compressibility.

\section{Conclusions}

TSDIA provides a systematic approach to the derivation of a transport model for weakly compressible turbulence. The occurrence of diffusivities for entropy and pressure and of shear and bulk viscosities acting on the mean flow are effects that could be anticipated by analogy with incompressible turbulence modeling. However, by incorporating the effects of wave motion into the description of compressible turbulence, we find Mach number-dependent modifications of the specific heat ratio and the speed of sound. TSDIA further predicts that terms dependent on gradients of turbulence quantities will appear in the mean flow equations. At this point, we have only given a preliminary account of these effects, but it is clear that they could not be predicted by other methods. 
Nonlinear effects, analogous to normal stress effects in simple shear flow, are also accessible to TSDIA analysis. The lengthy analyses required to evaluate such effects can be expedited by symbolic calculations. ${ }^{20}$ Other more straightforward extensions of this analysis are to compressible heat transfer modeling, gravitational effects, and effects of rotation on compressible turbulence. DIA and TSDIA provide a unified framework in which to analyze such coupled effects in a general and systematic manner.

Open issues include the accurate evaluation of turbulent gradient terms and the consideration of the effects of compressibility on the two equation model. It is evident that these effects will be of order $M_{t}^{2}$, but they should be evaluated for potential extension of this theory to strong compressibility.

\section{APPENDIX I. Outline of the DIA theory of passive fields coupled to incom- pressible turbulence}

Let a general system of fluctuating fields $q^{i}$ be coupled to incompressible turbulence by equations of the form

$$
\begin{aligned}
& \dot{q}^{i}(\mathbf{k}, t)+A_{k}^{i} q^{k}(\mathbf{k}, t)+\int_{\mathbf{k}=\mathbf{p}+\mathbf{q}} d \mathbf{p} d \mathbf{q}\left\{B_{k}^{i \mu}(\mathbf{k}, \mathbf{p}, \mathbf{q}) \mathbf{u}_{\mu}^{\infty}(\mathbf{p}, t)\right. \\
& \left.+C_{k}^{i}(\mathbf{k}, \mathbf{p}, \mathbf{q}) p^{\infty}(\mathbf{p}, t)\right\} q^{k}(\mathbf{q}, t)+\mathcal{A}_{k}^{i} q^{k}(\mathbf{k}, t)=0
\end{aligned}
$$

where $A$ represents linear couplings among the $q^{i}, B$ and $C$ couple the $q^{i}$ to incompressible turbulence, and $\mathcal{A}$ couples $q^{i}$ to external fields. DIA closes all moments of the $q^{i}$ in terms of the response matrix ${ }^{5}$

$$
G_{j}^{i}=\left\langle\delta q^{i} / \delta f^{j}>\right.
$$

where $f^{j}$ is a small perturbation added to the right side of the equation for $q^{j}$ in Eq. (101). DIA also provides a set of evolution equations for this matrix of the general form

$$
\begin{aligned}
& \dot{G}_{j}^{i}(\mathbf{k}, t, s)+A_{k}^{i} G_{j}^{k}(\mathbf{k}, t, s)+\mathcal{A}_{k}^{i} G_{j}^{k}(\mathbf{k}, t, s) \\
& +\int_{0}^{t} d r \eta_{p}^{i}(\mathbf{k}, t, r) G_{j}^{p}(\mathbf{k}, r, s)=\delta_{j}^{i} \delta(t-s)
\end{aligned}
$$

in which the damping factors $\eta$ are

$$
\begin{aligned}
& \eta_{p}^{i}(\mathbf{k}, t, s)=\int_{\mathbf{k}=\mathbf{p}+\mathbf{q}} d \mathbf{p} d \mathbf{q}\left\{B_{k}^{i \mu}(\mathbf{k}, \mathbf{p}, \mathbf{q}) B_{p}^{l \nu}(\mathbf{q},-\mathbf{p}, \mathbf{k}) G_{l}^{k}(\mathbf{p}, t, s) Q_{\mu \nu}(\mathbf{q}, t, s)\right. \\
& \left.+C_{k}^{i}(\mathbf{k}, \mathbf{p}, \mathbf{q}) C_{p}^{l}(\mathbf{q},-\mathbf{p}, \mathbf{k}) G_{l}^{k}(\mathbf{p}, t, s) Q^{p}(\mathbf{q}, t, s)\right\}
\end{aligned}
$$


Note that the damping factors $\eta$ depend on the various couplings through their dependence on the response functions.

Turbulence of the fields $q^{i}$ generated by some external agency is described by the inhomogeneous form of Eq. (101),

$$
\begin{aligned}
& \dot{q}^{i}(\mathbf{k}, t)+A_{k}^{i} q^{k}(\mathbf{k}, t)+\int_{\mathbf{k}=\mathbf{p}+\mathbf{q}} d \mathbf{p} d \mathbf{q}\left\{B_{k}^{i \mu}(\mathbf{k}, \mathbf{p}, \mathbf{q}) \mathbf{u}_{\mu}^{\infty}(\mathbf{p}, t) q^{k}(\mathbf{q}, t)\right. \\
& +C_{k}^{i}(\mathbf{k}, \mathbf{p}, \mathbf{q}) p^{\infty}(\mathbf{p}, t) q^{k}(\mathbf{q}, t)+\mathcal{A}_{k}^{i} q^{k}(\mathbf{k}, t)=F^{i}(\mathbf{k}, t)
\end{aligned}
$$

which has the formal solution

$$
q^{i}(\mathbf{k}, t)=\int_{0}^{t} d s G_{j}^{i}(\mathbf{k}, t, s) F^{j}(\mathbf{k}, s)
$$

It must be understood that Eq. (104) may only be applied to form correlations of the fluctuating fields $q^{i}$; DIA thus provides a statistical solution to Eq. (103), not a strict pointwise solution. It can be noted that DIA is the first in a sequence of successively more elaborate statistical approximations. ${ }^{29}$

Even the simplest equations of the general type of Eq. (102), such as the DIA equations for shear flow, ${ }^{19}$ seem to defy analysis. A simplification which makes some progress possible is to treat the external field couplings perturbatively. Thus, rewrite Eq. (101) as

$$
\dot{q}^{i}+A_{j}^{i} q^{j}+B_{k}^{i j} \mathbf{u}_{j}^{\infty} q^{k}+C_{j}^{i} p^{\infty} q^{j}=-\mathcal{A}_{j}^{i} q^{j}
$$

and treat the right side as a small forcing term. If the response matrix $G_{0 j}^{i}$ for the left side of Eq. (105) can be evaluated, then it is possible to construct a formal perturbation series in powers of $\mathcal{A}$ which satisfies Eq. (105): write

$$
q^{i}=q_{0}^{i}+q_{1}^{i}+\cdots
$$

where $q_{p}^{i}$ is of order $p$ in $\mathcal{A}$, then substitute in Eq. (105). The solution of the inhomogeneous system Eq. (103) is Eq. (106)with

$$
\begin{gathered}
q_{0}^{i}(\mathbf{k}, t)=\int_{0}^{t} d s G_{0 j}^{i}(\mathbf{k}, t, s) F^{j}(\mathbf{k}, s) \\
q_{1}^{i}(\mathbf{k}, t)=\int_{0}^{t} d s G_{0 j}^{i}(\mathbf{k}, t, s) q_{0}^{j}(\mathbf{k}, s) \\
\ldots \\
q_{n}^{i}(\mathbf{k}, t)=\int_{0}^{t} d s G_{0 j}^{i}(\mathbf{k}, t, s) q_{n-1}^{j}(\mathbf{k}, s)
\end{gathered}
$$


A particular perturbation expansion of this form was proposed by Leslie ${ }^{19}$ to solve the DIA shear flow equations. This expansion is essentially a Neumann series for the response matrix in powers of $\mathcal{A}$. TSDIA can also be understood in part as an expansion of this type.

It may prove difficult to evaluate the response matrix even from the left side of Eq. (105). The difficulties are caused more by coupling terms like $B$ and $C$ than by $A$, which can often be diagonalized. A simplification which carries the procedure leading from Eq. (101) to Eq. (105) one step further is to treat all couplings among the $q^{i}$ as perturbations. This defines the diagonal approximation in which the response functions are evaluated from the simplified system

$$
\dot{q}^{i}+A_{i}^{i} q^{i}+B_{i}^{i j} \mathbf{u}_{j}^{\infty} q^{i}+C_{i}^{i} p^{\infty} q^{i}=0 \text { no sum on } i
$$

As before, the couplings can be reintroduced perturbatively; however, it will not be necessary to write the expressions generated by this expansion explicitly. Note that any combination of coupling terms can be treated perturbatively in this fashion.

Applied to the system Eq. (105), the diagonal approximation will conceal an interesting physical property of compressible turbulence: the occurrence of wave motions. In order to reveal these motions, it is necessary to find an approximation scheme intermediate in complexity between the diagonal approximation and the full system of governing equations. The approximation represented by Eq. (28) and the approximation outlined in Sect. VI both have this property.

\section{APPENDIX II. List of correlations}

A. $\left\langle\mathbf{u}^{\infty} p\right\rangle$

$$
\begin{aligned}
& -<u_{i}^{\infty} p>=\int d \mathbf{k} \int_{0}^{t} d r \\
& \left\{+G^{p p}(\mathbf{k}, t, r)<u_{i}^{\infty}(-\mathbf{k}, t) \frac{\partial p^{\infty}}{\partial T}(\mathbf{k}, r)>\right. \\
& +G^{p p}(\mathbf{k}, t, r)<u_{i}^{\infty}(-\mathbf{k}, t) U_{p} \frac{\partial p^{\infty}}{\partial X_{p}}(\mathbf{k}, r)> \\
& +G^{p p}(\mathbf{k}, t, r)<u_{i}^{\infty}(-\mathbf{k}, t) U_{p} i k_{p} p^{\infty}(\mathbf{k}, r)> \\
& +G^{p p}(\mathbf{k}, t, r)<u_{i}^{\infty}(-\mathbf{k}, t) u_{j}^{\infty}(\mathbf{k}, r)>\frac{\partial P}{\partial X_{j}}
\end{aligned}
$$




$$
\begin{aligned}
& +G^{p p}(\mathbf{k}, t, r) \gamma<u_{i}^{\infty}(-\mathbf{k}, t) p^{\infty}(\mathbf{k}, r)>\frac{\partial U_{p}}{\partial X_{p}} \\
& +G^{p p}(\mathbf{k}, t, r)<u_{i}^{\infty}(-\mathbf{k}, t) \frac{\partial p^{\infty}}{\partial r}(\mathbf{k}, r)> \\
& +G^{p p}(\mathbf{k}, t, r) \int_{\mathbf{k}=\mathbf{p}+\mathbf{q}} d \mathbf{p} d \mathbf{q}<u_{i}^{\infty}(-\mathbf{k}, t) u_{j}^{\infty}(\mathbf{p}, r) i q_{j} p^{\infty}(\mathbf{q}, r)> \\
& +G^{p p}(\mathbf{k}, t, r) \int_{\mathbf{k}=\mathbf{p}+\mathbf{q}} d \mathbf{p} d \mathbf{q}<u_{i}^{\infty}(-\mathbf{k}, t) u_{j}^{\infty}(\mathbf{p}, r) \frac{\partial p^{\infty}}{\partial X_{j}}(\mathbf{q}, r)> \\
& -G_{j}^{p u}(\mathbf{k}, t, r) \frac{1}{c^{2} R^{2}} \int_{\mathbf{k}=\mathbf{p}+\mathbf{q}} d \mathbf{p} d \mathbf{q}<u_{i}^{\infty}(-\mathbf{k}, r) p^{\infty}(\mathbf{p}, r) i q_{j} p^{\infty}(\mathbf{q}, r)> \\
& -G_{j}^{p u}(\mathbf{k}, t, r) \frac{1}{c^{2} R^{2}} \int_{\mathbf{k}=\mathbf{p}+\mathbf{q}} d \mathbf{p} d \mathbf{q}<u_{i}^{\infty}(-\mathbf{k}, r) p^{\infty}(\mathbf{p}, r) \frac{\partial p^{\infty}}{\partial X_{j}}(\mathbf{q}, r)> \\
& -G_{j}^{p u}(\mathbf{k}, t, r) \frac{1}{c^{2} R^{2}}<u_{i}^{\infty}(-\mathbf{k}, t) p^{\infty}(\mathbf{k}, r)>\frac{\partial P}{\partial X_{j}} \\
& +G_{j}^{p u}(\mathbf{k}, t, r)<u_{i}^{\infty}(-\mathbf{k}, t) u_{m}^{\infty}(\mathbf{k}, r)>\left(\frac{\partial U_{j}}{\partial X_{m}}\right) C^{C}
\end{aligned}
$$

Several of these terms either vanish identically, or vanish to the present order of approximation. As the same arguments will apply to all subsequent correlations, we give the argument for each term.

Note first that $G_{j}^{p u}$ should be replaced by the scalar $G^{p \phi}$ using Eq. (31). The proportionality of $G_{j}^{p u}$ to $k_{j}$ causes several terms to vanish.

(a), (b) both vanish to this order: correlation $\left\langle\mathbf{u}^{\infty} p^{\infty}\right\rangle$ is zero in homogeneous turbulence; a nonvanishing contribution will require one additional gradient in $X, T$ leading to a higher order term. This argument also applies to terms $(\epsilon),(j),(k)$

(c) vanishes identically on integration over $\mathbf{k}$ because it changes sign under $\mathbf{k} \rightarrow-\mathbf{k}$. This argument also shows that $(l)$ vanishes.

$(d)$ is a gradient transport contribution analyzed below.

$(f)$ is a nonvanishing turbulence transport term discussed below. Other terms of this type are $(h)$ and $(i)$.

(g) vanishes since it is changes sign under the simultaneous transformation of wavevectors $\mathbf{p} \rightarrow-\mathbf{p}, \mathbf{q} \rightarrow-\mathbf{q}, \mathbf{k} \rightarrow-\mathbf{k}$. This transformation is admissible because it preserves the triangle condition $\mathbf{k}=\mathbf{p}+\mathbf{q}$. 
The nonvanishing terms are $(d),(f),(h),(i)$. Of these terms, $(d)$ contains a mean flow gradient, and $(f),(h),(i)$ will depend on gradients of turbulence quantities. Note that although $(f)$ vanishes to lowest order, it is possible that it makes a nonvanishing contribution at higher order in TSDIA. The resolution of this issue is left to further research. The terms $(h),(i)$ are triangle integral terms of a type also discussed by Yoshizawa. ${ }^{1}$

B. $\left\langle u_{i} p^{\infty}>\right.$

$$
\begin{aligned}
& -<u_{i} p^{\infty}>=\int d \mathbf{k} \int_{0}^{t} d r \\
& \left\{+G_{i}^{u p}(\mathbf{k}, t, r)<p^{\infty}(-\mathbf{k}, t) \frac{\partial p^{\infty}}{\partial T}(\mathbf{k}, r)>\right. \\
& +G_{i}^{u p}(\mathbf{k}, t, r)<p^{\infty}(-\mathbf{k}, t) U_{j} \frac{\partial p^{\infty}}{\partial X_{j}}(\mathbf{k}, r)> \\
& +G_{i}^{u p}(\mathbf{k}, t, r)<p^{\infty}(-\mathbf{k}, t) U_{j} i k_{j} p^{\infty}(\mathbf{k}, r)> \\
& +G_{i}^{u p}(\mathbf{k}, t, r)<p^{\infty}(-\mathbf{k}, t) u_{j}^{\infty}(\mathbf{k}, r)>\frac{\partial P}{\partial X_{j}} \\
& +G_{i}^{u p}(\mathbf{k}, t, r) \gamma<p^{\infty}(-\mathbf{k}, t) p^{\infty}(\mathbf{k}, r)>\frac{\partial U_{j}}{\partial X_{j}} \\
& +G_{i}^{u p}(\mathbf{k}, t, r)<p^{\infty}(-\mathbf{k}, t) \frac{\partial p^{\infty}}{\partial r}(\mathbf{k}, r)>\text {. } \\
& +G_{i}^{u p}(\mathbf{k}, t, r) \int_{\mathbf{k}=\mathbf{p}+\mathbf{q}} d \mathbf{p} d \mathbf{q}<p^{\infty}(-\mathbf{k}, t) u_{j}^{\infty}(\mathbf{p}, r) \frac{\partial p^{\infty}}{\partial X_{j}}(\mathbf{q}, r)> \\
& +G_{i}^{u p}(\mathbf{k}, t, r) \int_{\mathbf{k}=\mathbf{p}+\mathbf{q}} d \mathbf{p} d \mathbf{q}<p^{\infty}(-\mathbf{k}, t) u_{j}^{\infty}(\mathbf{p}, r) i q_{j} p^{\infty}(\mathbf{q}, r)> \\
& -G_{i j}^{u u}(\mathbf{k}, t, r) \frac{1}{c^{2} R^{2}} \int_{\mathbf{k}=\mathbf{p}+\mathbf{q}} d \mathbf{p} d \mathbf{q}<p^{\infty}(-\mathbf{k}, t) p^{\infty}(\mathbf{p}, r) i q_{j} p^{\infty}(\mathbf{q}, r)> \\
& -G_{i j}^{u u}(\mathbf{k}, t, r) \frac{1}{c^{2} R^{2}} \int_{\mathbf{k}=\mathbf{p}+\mathbf{q}} d \mathbf{p} d \mathbf{q}<p^{\infty}(-\mathbf{k}, t) p^{\infty}(\mathbf{p}, r) \frac{\partial p^{\infty}}{\partial X_{j}}(\mathbf{q}, r)> \\
& -G_{i j}^{u u}(\mathbf{k}, t, r) \frac{1}{c^{2} R^{2}}<p^{\infty}(-\mathbf{k}, t) p^{\infty}(\mathbf{k}, r)>\frac{\partial P}{\partial X_{j}} \\
& \left.+G_{i j}^{u u}(\mathbf{k}, t, r)<p^{\infty}(-\mathbf{k}, t) u_{p}^{\infty}(\mathbf{k}, r)>\left(\frac{\partial U_{j}}{\partial X_{p}}\right)^{C}\right\}
\end{aligned}
$$

Arguments like those used in case A show that the nonvanishing terms are $(c),(h),(j),(k)$. Of these, only $(k)$ depends explicitly on the mean flow.

C. $\left\langle\mathbf{u} \cdot \nabla p^{\infty}\right\rangle$

It will be convenient to separate the terms containing $i k_{i}$ and the terms containing $\partial / \partial X_{i}$, which will be denoted by the subscripts $x$ and $X$ respectively. This gives for the 
terms containing $i k_{i}$

$$
\begin{aligned}
& -<\mathbf{u} \cdot \nabla_{x} p^{\infty}>=\int d \mathbf{k} \int_{0}^{t} d r \\
& \left\{+G_{i}^{u p}(\mathbf{k}, t, r)<-i k_{i} p^{\infty}(-\mathbf{k}, t) \frac{\partial p^{\infty}}{\partial T}(\mathbf{k}, r)>\right. \\
& +G_{i}^{u p}(\mathbf{k}, t, r)<-i k_{i} p^{\infty}(-\mathbf{k}, t) U_{j} \frac{\partial p^{\infty}}{\partial X_{j}}(\mathbf{k}, r)> \\
& +G_{i}^{u p}(\mathbf{k}, t, r)<-i k_{i} p^{\infty}(-\mathbf{k}, t) U_{j} i k_{j} p^{\infty}(\mathbf{k}, r)> \\
& +G_{i}^{u p}(\mathbf{k}, t, r)<-i k_{i} p^{\infty}(-\mathbf{k}, t) u_{j}^{\infty}(\mathbf{k}, r)>\frac{\partial P}{\partial X_{j}} \\
& +G_{i}^{u p}(\mathbf{k}, t, r) \gamma<-i k_{i} p^{\infty}(-\mathbf{k}, t) p^{\infty}(\mathbf{k}, r)>\frac{\partial U_{j}}{\partial X_{j}} \\
& +G_{i}^{u p}(\mathbf{k}, t, r)<-i k_{i} p^{\infty}(-\mathbf{k}, t) \frac{\partial p^{\infty}}{\partial r}(\mathbf{k}, r)> \\
& +G_{i}^{u p}(\mathbf{k}, t, r) \int_{\mathbf{k}=\mathbf{p}+\mathbf{q}} d \mathbf{p} d \mathbf{q}<-i k_{i} p^{\infty}(-\mathbf{k}, t) u_{j}^{\infty}(\mathbf{p}, r) \frac{\partial p^{\infty}}{\partial X_{j}}(\mathbf{q}, r)> \\
& +G_{i}^{u p}(\mathbf{k}, t, r) \int_{\mathbf{k}=\mathbf{p}+\mathbf{q}} d \mathbf{p} d \mathbf{q}<-i k_{i} p^{\infty}(-\mathbf{k}, t) u_{j}^{\infty}(\mathbf{p}, r) i q_{j} p^{\infty}(\mathbf{q}, r)> \\
& -G_{i j}^{u u}(\mathbf{k}, t, r) \frac{1}{c^{2} R^{2}} \int_{\mathbf{k}=\mathbf{p}+\mathbf{q}} d \mathbf{p} d \mathbf{q}<-i k_{i} p^{\infty}(-\mathbf{k}, t) p^{\infty}(\mathbf{p}, r) i q_{j} p^{\infty}(\mathbf{q}, r)> \\
& -G_{i j}^{u u}(\mathbf{k}, t, r) \frac{1}{c^{2} R^{2}} \int_{\mathbf{k}=\mathbf{p}+\mathbf{q}} d \mathbf{p} d \mathbf{q}<-i k_{i} p^{\infty}(-\mathbf{k}, t) p^{\infty}(\mathbf{p}, r) \frac{\partial p^{\infty}}{\partial X_{j}}(\mathbf{q}, r)> \\
& -G_{i j}^{u u}(\mathbf{k}, t, r) \frac{1}{c^{2} R^{2}}<-i k_{i} p^{\infty}(-\mathbf{k}, t) p^{\infty}(\mathbf{k}, r)>\frac{\partial P}{\partial X_{j}} \\
& \left.+G_{i j}^{u u}(\mathbf{k}, t, r)<-i k_{i} p^{\infty}(-\mathbf{k}, t) u_{p}^{\infty}(\mathbf{k}, r)>\left(\frac{\partial U_{j}}{\partial X_{p}}\right)^{C}\right\}
\end{aligned}
$$

The nonvanishing correlations are $(a),(b),(e),(f),(g),(j)$. Of these, only $(e)$ depends explicitly on the mean flow.

The terms corresponding to those of Eq. (109) in which $i k_{i}$ is replaced by $\partial / \partial X_{i}$ are

$$
\begin{aligned}
-<\mathbf{u} \cdot \nabla_{X} p^{\infty} & =\int d \mathbf{k} \int_{0}^{t} d r \\
\left\{+G_{i}^{u p}(\mathbf{k}, t, r)\right. & <\frac{\partial p^{\infty}}{\partial X_{i}}(-\mathbf{k}, t) \frac{\partial p^{\infty}}{\partial T}(\mathbf{k}, r)> \\
+G_{i}^{u p}(\mathbf{k}, t, r) & <\frac{\partial p^{\infty}}{\partial X_{i}}(-\mathbf{k}, t) U_{j} \frac{\partial p^{\infty}}{\partial X_{j}}(\mathbf{k}, r)> \\
+G_{i}^{u p}(\mathbf{k}, t, r) & <\frac{\partial p^{\infty}}{\partial X_{i}}(-\mathbf{k}, t) U_{i} i k_{j} p^{\infty}(\mathbf{k}, r)>
\end{aligned}
$$




$$
\begin{aligned}
& +G_{i}^{u p}(\mathbf{k}, t, r)<\frac{\partial p^{\infty}}{\partial X_{i}}(-\mathbf{k}, t) u_{j}^{\infty}(\mathbf{k}, r)>\frac{\partial P}{\partial X_{j}} \\
& +G_{i}^{u p}(\mathbf{k}, t, r) \gamma<\frac{\partial p^{\infty}}{\partial X_{i}}(-\mathbf{k}, t) p^{\infty}(\mathbf{k}, r)>\frac{\partial U_{j}}{\partial X_{j}} \\
& +G_{i}^{u p}(\mathbf{k}, t, r)<\frac{\partial p^{\infty}}{\partial X_{i}}(-\mathbf{k}, t) \frac{\partial p^{\infty}}{\partial r}(\mathbf{k}, r)> \\
& +G_{i}^{u p}(\mathbf{k}, t, r) \int_{\mathbf{k}=\mathbf{p}+\mathbf{q}} d \mathbf{p} d \mathbf{q}<\frac{\partial p^{\infty}}{\partial X_{i}}(-\mathbf{k}, t) u_{j}^{\infty}(\mathbf{p}, r) \frac{\partial p^{\infty}}{\partial X_{j}}(\mathbf{q}, r)> \\
& +G_{i}^{u p}(\mathbf{k}, t, r) \int_{\mathbf{k}=\mathbf{p}+\mathbf{q}} d \mathbf{p} d \mathbf{q}<\frac{\partial p^{\infty}}{\partial X_{i}}(-\mathbf{k}, t) u_{j}^{\infty}(\mathbf{p}, r) i q_{j} p^{\infty}(\mathbf{q}, r)> \\
& -G_{i j}^{u u}(\mathbf{k}, t, r) \frac{1}{c^{2} R^{2}} \int_{\mathbf{k}=\mathbf{p}+\mathbf{q}} d \mathbf{p} d \mathbf{q}<\frac{\partial p^{\infty}}{\partial X_{i}}(-\mathbf{k}, t) p^{\infty}(\mathbf{p}, r) i q_{j} p^{\infty}(\mathbf{q}, r)> \\
& -G_{i j}^{u u}(\mathbf{k}, t, r) \frac{1}{c^{2} R^{2}} \int_{\mathbf{k}=\mathbf{p}+\mathbf{q}} d \mathbf{p} d \mathbf{q}<\frac{\partial p^{\infty}}{\partial X_{i}}(-\mathbf{k}, t) p^{\infty}(\mathbf{p}, r) \frac{\partial p^{\infty}}{\partial X_{j}}(\mathbf{q}, r)> \\
& -G_{i j}^{u u}(\mathbf{k}, t, r) \frac{1}{c^{2} R^{2}}<\frac{\partial p^{\infty}}{\partial X_{i}}(-\mathbf{k}, t) p^{\infty}(\mathbf{k}, r)>\frac{\partial P}{\partial X_{j}} \\
& \left.+G_{i j}^{u u}(\mathbf{k}, t, r)<\frac{\partial p^{\infty}}{\partial X_{i}}(-\mathbf{k}, t) u_{p}^{\infty}(\mathbf{k}, r)>\left(\frac{\partial U_{j}}{\partial X_{p}}\right)^{C}\right\}
\end{aligned}
$$

In determining which terms are to be retained, it must be noted that, unlike previous correlations, there is no leading derivative with respect to $X$. Therefore, terms containing up to two derivatives with respect to $X$ and $T$ must be retained. Therefore, the nonvanishing terms are $(c),(h),(j),(k)$.

D. $\left\langle\mathbf{u}^{\infty} \mathbf{u}\right\rangle$

$$
\begin{aligned}
& -<u_{i}^{\infty} u_{j}>=\int d \mathbf{k} \int_{0}^{t} d r \\
& \left\{-G_{j m}^{u u}(\mathbf{k}, t, r) \frac{1}{c^{2} R^{2}} \int_{\mathbf{k}=\mathbf{p}+\mathbf{q}} d \mathbf{p} d \mathbf{q}<u_{i}^{\infty}(-\mathbf{k}, t) p^{\infty}(\mathbf{p}, r) i q_{m} p^{\infty}(\mathbf{q}, r)>\right. \\
& -G_{j m}^{u u}(\mathbf{k}, t, r) \frac{1}{c^{2} R^{2}} \int_{\mathbf{k}=\mathbf{p}+\mathbf{q}} d \mathbf{p} d \mathbf{q}<u_{i}^{\infty}(-\mathbf{k}, t) p^{\infty}(\mathbf{p}, r) \frac{\partial p^{\infty}}{\partial X_{m}}(\mathbf{q}, r)> \\
& -G_{j m}^{u u}(\mathbf{k}, t, r) \frac{1}{c^{2} R^{2}}<u_{i}^{\infty}(-\mathbf{k}, t) p^{\infty}(\mathbf{k}, r)>\frac{\partial P}{\partial X_{m}} \\
& +G_{j m}^{u u}(\mathbf{k}, t, r)<u_{i}^{\infty}(-\mathbf{k}, t) u_{p}^{\infty}(\mathbf{k}, r)>\left(\frac{\partial U_{m}}{\partial X_{p}}\right)^{C} \\
& +G_{j}^{u p}(\mathbf{k}, t, r)<u_{i}^{\infty}(-\mathbf{k}, t) \frac{\partial p^{\infty}}{\partial T}(\mathbf{k}, r)> \\
& +G_{j}^{u p}(\mathbf{k}, t, r)<u_{i}^{\infty}(-\mathbf{k}, t) U_{p} \frac{\partial p^{\infty}}{\partial X_{p}}(\mathbf{k}, r)>
\end{aligned}
$$




$$
\begin{aligned}
& +G_{j}^{u p}(\mathbf{k}, t, r)<u_{i}^{\infty}(-\mathbf{k}, t) U_{p} i k_{p} p^{\infty}(\mathbf{k}, r)> \\
& +G_{j}^{u p}(\mathbf{k}, t, r)<u_{i}^{\infty}(-\mathbf{k}, t) u_{m}^{\infty}(\mathbf{k}, r)>\frac{\partial P}{\partial X_{m}} \\
& +G_{j}^{u p}(\mathbf{k}, t, r) \gamma<u_{i}^{\infty}(-\mathbf{k}, t) p^{\infty}(\mathbf{k}, r)>\frac{\partial U_{p}}{\partial X_{p}} \\
& +G_{j}^{u p}(\mathbf{k}, t, r)<u_{i}^{\infty}(-\mathbf{k}, t) \frac{\partial p^{\infty}}{\partial r}(\mathbf{k}, r)> \\
& +G_{j}^{u p}(\mathbf{k}, t, r) \int_{\mathbf{k}=\mathbf{p}+\mathbf{q}} d \mathbf{p} d \mathbf{q}<u_{i}^{\infty}(-\mathbf{k}, t) u_{m}^{\infty}(\mathbf{p}, r) i q_{m} p^{\infty}(\mathbf{q}, r)> \\
& \left.+G_{j}^{u p}(\mathbf{k}, t, r) \int_{\mathbf{k}=\mathbf{p}+\mathbf{q}} d \mathbf{p} d \mathbf{q}<u_{i}^{\infty}(-\mathbf{k}, t) u_{m}^{\infty}(\mathbf{p}, r) \frac{\partial p^{\infty}}{\partial X_{m}}(\mathbf{q}, r)>\right\}
\end{aligned}
$$

The nonvanishing terms are $(b),(d),(g),(k)$. Of these, only $(d)$ depends explicitly on the mean flow.

E. $\left\langle\mathbf{u} \cdot \nabla \mathbf{u}^{\infty}>\right.$

Again writing the terms in $i k_{j}$ and $\partial / \partial X_{j}$ separately, denoting them respectively by subscripts $x$ and $X$, the terms in $i k_{j}$ are

$$
\begin{aligned}
-< & \left(\mathbf{u} \cdot \nabla_{x} \mathbf{u}^{\infty}\right)_{i}>=\int d \mathbf{k} \int_{0}^{t} d r \\
\{ & -G_{j m}^{u u}(\mathbf{k}, t, r) \frac{1}{c^{2} R^{2}} \int_{\mathbf{k}=\mathbf{p}+\mathbf{q}} d \mathbf{p} d \mathbf{q}<-i k_{j} u_{i}^{\infty}(-\mathbf{k}, t) p^{\infty}(\mathbf{p}, r) i q_{m} p^{\infty}(\mathbf{q}, r)> \\
& -G_{j m}^{u u}(\mathbf{k}, t, r) \frac{1}{c^{2} R^{2}} \int_{\mathbf{k}=\mathbf{p}+\mathbf{q}} d \mathbf{p} d \mathbf{q}<-i k_{j} u_{i}^{\infty}(-\mathbf{k}, t) p^{\infty}(\mathbf{p}, r) \frac{\partial p^{\infty}}{\partial X_{m}}(\mathbf{q}, r)> \\
& -G_{j m}^{u u}(\mathbf{k}, t, r) \frac{1}{c^{2} R^{2}}<-i k_{j} u_{i}^{\infty}(-\mathbf{k}, t) p^{\infty}(\mathbf{k}, r)>\frac{\partial P}{\partial X_{m}} \\
& +G_{j m}^{u u}(\mathbf{k}, t, r)<-i k_{j} u_{i}^{\infty}(-\mathbf{k}, t) u_{p}^{\infty}(\mathbf{k}, r)>\left(\frac{\partial U_{m}}{\partial X_{p}}\right)^{C} \\
& +G_{j}^{u p}(\mathbf{k}, t, r)<-i k_{j} u_{i}^{\infty}(-\mathbf{k}, t) \frac{\partial p^{\infty}}{\partial T}(\mathbf{k}, r)> \\
& +G_{j}^{u p}(\mathbf{k}, t, r)<-i k_{j} u_{i}^{\infty}(-\mathbf{k}, t) U_{p} \frac{\partial p^{\infty}}{\partial X_{p}}(\mathbf{k}, r)> \\
& +G_{j}^{u p}(\mathbf{k}, t, r)<-i k_{j} u_{i}^{\infty}(-\mathbf{k}, t) U_{p} i k_{p} p^{\infty}(\mathbf{k}, r)> \\
+ & G_{j}^{u p}(\mathbf{k}, t, r)<-i k_{j} u_{i}^{\infty}(-\mathbf{k}, t) u_{m}^{\infty}(\mathbf{k}, r)>\frac{\partial P}{\partial X_{m}} \\
+ & G_{j}^{u p}(\mathbf{k}, t, r) \gamma<-i k_{j} u_{i}^{\infty}(-\mathbf{k}, t) p^{\infty}(\mathbf{k}, r)>\frac{\partial U_{p}}{\partial X_{p}} \\
+ & G_{j}^{u p}(\mathbf{k}, t, r)<-i k_{j} u_{i}^{\infty}(-\mathbf{k}, t) \frac{\partial p^{\infty}}{\partial r}(\mathbf{k}, r)>
\end{aligned}
$$




$$
\begin{aligned}
& +G_{j}^{u p}(\mathbf{k}, t, r) \int_{\mathbf{k}=\mathbf{p}+\mathbf{q}} d \mathbf{p} d \mathbf{q}<-i k_{j} u_{i}^{\infty}(-\mathbf{k}, t) u_{m}^{\infty}(\mathbf{p}, r) i q_{m} p^{\infty}(\mathbf{q}, r)> \\
& \left.+G_{j}^{u p}(\mathbf{k}, t, r) \int_{\mathbf{k}=\mathbf{p}+\mathbf{q}} d \mathbf{p} d \mathbf{q}<-i k_{j} u_{i}^{\infty}(-\mathbf{k}, t) u_{m}^{\infty}(\mathbf{p}, r) \frac{\partial p^{\infty}}{\partial X_{m}}(\mathbf{q}, r)>\right\}
\end{aligned}
$$

The nonvanishing terms are $(a),(e),(f),(h),(i),(j),(l)$. Of these, $(h)$ depends explicitly on the mean field.

The terms dependent on $\partial / \partial X_{j}$ are

$$
\begin{aligned}
-< & \left(\mathbf{u} \cdot \nabla_{X} \mathbf{u}^{\infty}\right)_{i j}>=-\int d \mathbf{k} \int_{0}^{t} d r \\
\{ & -G_{j m}^{u u}(\mathbf{k}, t, r) \frac{1}{c^{2} R^{2}} \int_{\mathbf{k}=\mathbf{p}+\mathbf{q}} d \mathbf{p} d \mathbf{q}<\frac{\partial u_{i}^{\infty}}{\partial X_{j}}(-\mathbf{k}, t) p^{\infty}(\mathbf{p}, r) i q_{m} p^{\infty}(\mathbf{q}, r)> \\
& -G_{j m}^{u u}(\mathbf{k}, t, r) \frac{1}{c^{2} R^{2}} \int_{\mathbf{k}=\mathbf{p}+\mathbf{q}} d \mathbf{p} d \mathbf{q}<\frac{\partial u_{i}^{\infty}}{\partial X_{j}}(-\mathbf{k}, t) p^{\infty}(\mathbf{p}, r) \frac{\partial p^{\infty}}{\partial X_{m}}(\mathbf{q}, r)> \\
& -G_{j m}^{u u}(\mathbf{k}, t, r) \frac{1}{c^{2} R^{2}}<\frac{\partial u_{i}^{\infty}}{\partial X_{j}}(-\mathbf{k}, t) p^{\infty}(\mathbf{k}, r)>\frac{\partial P}{\partial X_{m}} \\
& +G_{j m}^{u u}(\mathbf{k}, t, r)<\frac{\partial u_{i}^{\infty}}{\partial X_{j}}(-\mathbf{k}, t) u_{p}^{\infty}(\mathbf{k}, r)>\left(\frac{\partial U_{m}}{\partial X_{p}}\right)^{C} \\
& +G_{j}^{u p}(\mathbf{k}, t, r)<\frac{\partial u_{i}^{\infty}}{\partial X_{j}}(-\mathbf{k}, t) \frac{\partial p^{\infty}}{\partial T}(\mathbf{k}, r)> \\
& +G_{j}^{u p}(\mathbf{k}, t, r)<\frac{\partial u_{i}^{\infty}}{\partial X_{j}}(-\mathbf{k}, t) U_{p} \frac{\partial p^{\infty}}{\partial X_{p}}(\mathbf{k}, r)> \\
& +G_{j}^{u p}(\mathbf{k}, t, r)<\frac{\partial u_{i}^{\infty}}{\partial X_{j}}(-\mathbf{k}, t) U_{p} i k_{p} p^{\infty}(\mathbf{k}, r)> \\
& +G_{j}^{u p}(\mathbf{k}, t, r)<\frac{\partial u_{i}^{\infty}}{\partial X_{j}}(-\mathbf{k}, t) u_{m}^{\infty}(\mathbf{k}, r)>\frac{\partial P}{\partial X_{m}} \\
& +G_{j}^{u p}(\mathbf{k}, t, r) \gamma<\frac{\partial u_{i}^{\infty}}{\partial X_{j}}(-\mathbf{k}, t) p^{\infty}(\mathbf{k}, r)>\frac{\partial U_{p}}{\partial X_{p}} \\
& +G_{j}^{u p}(\mathbf{k}, t, r)<\frac{\partial u_{i}^{\infty}}{\partial X_{j}}(-\mathbf{k}, t) \frac{\partial p^{\infty}}{\partial r}(\mathbf{k}, t)> \\
& +G_{j}^{u p}(\mathbf{k}, t, r) \int_{\mathbf{k}=\mathbf{p}+\mathbf{q}} d \mathbf{p} d \mathbf{q}<\frac{\partial u_{i}^{\infty}}{\partial X_{j}}(-\mathbf{k}, t) u_{m}^{\infty}(\mathbf{p}, r) i q_{m} p^{\infty}(\mathbf{q}, r)> \\
& \left.+G_{j}^{u p}(\mathbf{k}, t, r) \int_{\mathbf{k}=\mathbf{p}+\mathbf{q}} d \mathbf{p} d \mathbf{q}<\frac{\partial u_{i}^{\infty}}{\partial X_{j}}(-\mathbf{k}, t) u_{m}^{\infty}(\mathbf{p}, r) \frac{\partial p^{\infty}}{\partial X_{m}}(\mathbf{q}, r)>\right\} \\
&
\end{aligned}
$$

Again note the action of $\partial / \partial X_{j}$ on $u_{i}^{\infty}(-\mathbf{k})$ only. The nonvanishing terms are $(b),(d),(g),(k)$. Of these, $(d)$ depends explicitly on the mean field. 
F. $\left\langle p p^{\infty}>\right.$

$$
\begin{aligned}
& -<p p^{\infty}>=\int d \mathbf{k} \int_{0}^{t} d r \\
& \left\{+G^{p p}(\mathbf{k}, t, r)<p^{\infty}(-\mathbf{k}, t) \frac{\partial p^{\infty}}{\partial T}(\mathbf{k}, r)>\right. \\
& +G^{p p}(\mathbf{k}, t, r)<p^{\infty}(-\mathbf{k}, t) U_{j} \frac{\partial p^{\infty}}{\partial X_{j}}(\mathbf{k}, r)> \\
& +G^{p p}(\mathbf{k}, t, r)<p^{\infty}(-\mathbf{k}, t) U_{j} i k_{j} p^{\infty}(\mathbf{k}, r)> \\
& +G^{p p}(\mathbf{k}, t, r)<p^{\infty}(-\mathbf{k}, t) u_{j}^{\infty}(\mathbf{k}, r)>\frac{\partial P}{\partial X_{j}} \\
& +G^{p p}(\mathbf{k}, t, r) \gamma<p^{\infty}(-\mathbf{k}, t) p^{\infty}(\mathbf{k}, r)>\frac{\partial U_{j}}{\partial X_{j}} \\
& +G^{p p}(\mathbf{k}, t, r)<p^{\infty}(-\mathbf{k}, t) \frac{\partial p^{\infty}}{\partial r}(\mathbf{k}, r)> \\
& +G^{p p}(\mathbf{k}, t, r) \int_{\mathbf{k}=\mathbf{p}+\mathbf{q}} d \mathbf{p} d \mathbf{q}<p^{\infty}(-\mathbf{k}, t) u_{j}^{\infty}(\mathbf{p}, r) \frac{\partial p^{\infty}}{\partial X_{j}}(\mathbf{q}, r)> \\
& +G^{p p}(\mathbf{k}, t, r) \int_{\mathbf{k}=\mathbf{p}+\mathbf{q}} d \mathbf{p} d \mathbf{q}<p^{\infty}(-\mathbf{k}, t) u_{j}^{\infty}(\mathbf{p}, r) i q_{j} p^{\infty}(\mathbf{q}, r)> \\
& -G_{j}^{p u}(\mathbf{k}, t, r) \frac{1}{c^{2} R^{2}} \int_{\mathbf{k}=\mathbf{p}+\mathbf{q}} d \mathbf{p} d \mathbf{q}<p^{\infty}(-\mathbf{k}, t) p^{\infty}(\mathbf{p}, r) i q_{j} p^{\infty}(\mathbf{q}, r)> \\
& -G_{j}^{p u}(\mathbf{k}, t, r) \frac{1}{c^{2} R^{2}} \int_{\mathbf{k}=\mathbf{p}+\mathbf{q}} d \mathbf{p} d \mathbf{q}<p^{\infty}(-\mathbf{k}, t) p^{\infty}(\mathbf{p}, r) \frac{\partial p^{\infty}}{\partial X_{j}}(\mathbf{q}, r)> \\
& -G_{j}^{p u}(\mathbf{k}, t, r) \frac{1}{c^{2} R^{2}}<p^{\infty}(-\mathbf{k}, t) p^{\infty}(\mathbf{k}, r)>\frac{\partial P}{\partial X_{j}} \\
& \left.+G_{j}^{p u}(\mathbf{k}, t, r)<p^{\infty}(-\mathbf{k}, t) u_{p}^{\infty}(\mathbf{k}, r)>\left(\frac{\partial U_{j}}{\partial X_{p}}\right)^{C}\right\}
\end{aligned}
$$

The nonvanishing terms are $(a),(b),(e),(f),(g),(j)$. Of these, $(e)$ depends explicitly on the mean fields.

\section{Appendix III. Approximate evaluation of turbulent correlation terms}

The evaluation of correlations which depend on gradients of turbulence quantities in Eqs. (108)-(112) is more elaborate. In general, it is necessary to replace $p^{\infty}$ by $\mathbf{u}^{\infty}$ using the Poisson equation and apply the quasinormal hypothesis to close the resulting moments of the velocity field. Provided that the integrals converge, it is possible to determine in advance at least the structure of the resulting single point moments. Since it will be useful to have a preliminary indication of the type of model which results, the form of these terms 
will be derived here, limiting attention to those terms which can be evaluated at the lowest order in TSDIA. Shimomura's result Eq. (52) is obtained by such higher order analysis, and reference can be made to Ref. 20 for the required procedure.

\section{A. Terms added to mean pressure equation}

Moments from Eq. (107):

(f) vanishes to lowest order since there are no isotropic functions of $\mathbf{u}^{\infty}$.

$(h) \sim \mathcal{O}\left(\nabla, K^{4}, \varepsilon^{-1}, c^{-2}\right)$ where the notation indicates only the total power of each factor in the result.

(i) vanishes to lowest order since it is of odd order in $\mathbf{u}^{\infty}$.

Moments from Eq. (108):

(c) vanishes to lowest order since there is no isotropic invariant of $\mathbf{U}$.

(h) vanishes like Eq. $(107 f)$.

(j) $\sim \mathcal{O}\left(\nabla, c^{-2}, K^{-4}, \varepsilon^{-1}\right)$

Moments from Eq. (109):

$(a),(b) \sim \mathcal{O}\left(D / D T, H^{-5 / 2}, c^{-2}\right)$

(f) vanishes to lowest order

$(g) \sim \mathcal{O}\left(\nabla, c^{-2}, H^{-4}, \varepsilon^{-1}\right)$

(i) $\sim I^{-} \varepsilon c^{-2}$

Moments from Eq. (110):

(c) vanishes to lowest order since there is no isotropic invariant of $\mathbf{U}$.

(h) vanishes to lowest order

$(j) \sim \mathcal{O}\left(\nabla^{2}, \Pi^{-4}, \varepsilon^{-1}, c^{-2}\right)$

Term $(k)$ is of a special type since it contains a mean velocity gradient, but its coefficient depends on gradients of turbulence quantities. The structure of this term is $(k) \sim\left[\mathcal{O}\left(\nabla, \Pi^{-3}, \varepsilon^{-1}, c^{-2}\right)\right] \nabla P$.

The mean pressure equation, with these effects added, has the form

$$
\begin{aligned}
& \frac{\partial P}{\partial t}+\mathbf{U} \cdot \nabla P+\gamma P \nabla \cdot \mathbf{U}=\nabla \cdot\left(\nu^{p p} \nabla P\right)-(\gamma-1) \Pi \nabla \cdot \mathbf{U} \\
& C_{1} c^{-2} K \varepsilon+C_{2} \mathcal{O}\left(\frac{D}{D T} \varepsilon\right)+C_{3} \mathcal{O}\left(\nabla, \nabla, K^{4} \varepsilon^{-1} c^{-2}\right)
\end{aligned}
$$


where the constants $C_{1}, C_{2}, C_{3}$ can be evaluated theoretically by evaluating the relevant integrals. The term containing a convective derivative occurs in the higher order TSDIA analysis of incompressible turbulence.

\section{B. Terms added to mean velocity equation}

Moments from Eq. (111):

(b) vanishes to lowest order

(g) vanishes to lowest order

(k) $\sim \mathbb{K}^{-2} c^{-2}$

Moments from Eq. (112):

(a) vanishes to lowest order.

(e) vanishes to lowest order.

(f) vanishes to lowest order.

(i) vanishes to lowest order.

(j) $\sim K^{-1 / 2} \varepsilon c^{-2}$

(l) $\sim \mathcal{O}\left(\nabla, K^{-2}, c^{-2}\right)$

Moments from Eq. (113):

(b) vanishes to lowest order.

(g) vanishes to lowest order.

(k) $\sim \mathcal{O}\left(\nabla, K^{-2}, c^{-2}\right)$

Term $(d)$, like $(110 k)$ depends on mean velocity gradients. It has the form (d) $\sim\left[\mathcal{O}\left(\nabla, K^{-2}, \varepsilon^{-1}\right)\right] \nabla \cdot \mathbf{U}$

Moments from Eq. (114):

$(a),(b) \sim D / D T\left(I^{-4} \varepsilon^{-1} c^{-2}\right)$

(f) vanishes to lowest order.

(g) vanishes to lowest order.

(j) $\sim \mathcal{O}\left(\nabla, K^{-9 / 2}, \varepsilon^{-1}, c^{-2}\right)$

Note that this group of terms appears with an additional factor of $c^{-2}$ and are therefore of higher order in $M_{t}$. 
These terms modify the mean velocity equation as follows:

$$
\begin{aligned}
& \frac{\partial \mathbf{U}}{\partial t}+\mathbf{U} \cdot \nabla \mathbf{U}+\frac{1}{R} \nabla P=\nabla \cdot\left\{-\frac{2}{3} K \mathbf{I}+\nu\left[\nabla \mathbf{U}+(\nabla \mathbf{U})^{T}\right]^{S}\right. \\
& +\nabla\left(\nu^{u u} \nabla \cdot \mathbf{U}\right)+\frac{1}{\hat{R}} \nabla P \\
& +C_{4} \nabla K^{-1 / 2} \varepsilon c^{-2}+C_{5} \mathcal{O}\left(\nabla^{2}, K^{-2}, c^{-2}\right)+C_{6} \mathcal{O}\left(\nabla, K^{-2}, \varepsilon^{-1}\right) \nabla \cdot U
\end{aligned}
$$

As before, the constants $C_{4}, C_{5}, C_{6}$ are obtained by evaluating the relevant integrals.

\section{References}

1. A. Yoshizawa, "Statistical analysis of the deviation of the Reynolds stress from its eddy viscosity representation," Phys. Fluids, 27, 1377 (1984).

2. G. P. Zank and W. H. Matthaeus, "The equations of nearly incompressible fluids I:Hydrodynamics, turbulence, and waves," Phys. Fluids A 3, 69 (1991).

3. G. Erlebacher, M. Y. Hussaini, H. O. Kreiss, and S. Sarkar, "The analysis and simulation of compressible turbulence," Theor. Comput. Fluid Dyn. 2, 73 (1990).

4. A. Yoshizawa, "Statistical analysis of compressible turbulent shear flow with special emphasis on turbulence modeling," Phys. Rev. A 46, 3292 (1992).

5. R. H. Kraichnan, "The structure of turbulence at very high Reynolds number," J. Fluid Mech., 5, 497 (1959).

6. J. P. Bertoglio, F. Bataille, and J. D. Marion, "Two point closures for weakly compressible turbulence," submitted to Phys. Fluids (1996).

7. G. J. Hartke, V. Canuto, and C. Alonso, "A DIA treatment of turbulence in a compressible fluid, I: Formalism," Phys. Fluids 31, 1034 (1988).

8. I. Staroselski, V. Yakhot, S. A. Orszag, and S. Kida, "Long time large scale properties of a randomly stirred compressible fluid," Phys. Rev. Lett. 65, 171 (1990).

9. F. Bataille, J. P. Bertoglio, and J. D. Marion, "Etude spectrale d'une turbulence isotrope faiblement compressible," C. R. Acad. Sci. Paris Ser II 315. 1459 (1992).

10. Y. Shimomura, "Theoretical modeling of thermally-driven turbulent flows," International Conference on Turbulent Heat Transfer, San Diego (1996).

11. H. Mlaouah, T. Tsuji, and Y. Nagano, "Discussion of laminar-turbulent transition of thermally induced flows in a square cavity," International Heat Transfer Conference, San Diego (1996). 
12. S. Girimaji, private communication.

13. P. G. Huang, G. N. Coleman, and P. Bradshaw, "Compressible turbulent channel flows: DNS results and modeling," J. Fluid Mech. 305, 185 (1996).

14. J. R. Ristorcelli, "A pseudo-sound constitutive relationship for the dilatational covariances in compressible turbulence: an analytical theory," ICASE Report 95-22 (1955).

15. S. Sarkar, G. Erlebacher, M. Y. Hussaini, and H. O. Kreiss, "The analysis and modeling of dilatational terms in compressible turbulence," J. Fluid Mech. 227, 473 (1991).

16. A. Mahalov, private communication.

17. F. Bataille, Y. Zhou, and J. P. Bertoglio, "Energy transfer in compressible turbulence," ICASE report 95-65 (1995).

18. R. H. Kraichnan, "Direct interaction approximation for shear and thermally driven turbulence," Phys. Fluids 7, 1048 (1964).

19. D. C. Leslie, Modern developments in the theory of turbulence, Oxford University Press (1972).

20. Y. Shimomura, "Theoretical study of triple velocity and pressure-velocity correlations in turbulent flow," Hiyoshi Rev. of Natural Science, 16, 12 (1994).

21. G. K. Batchelor, "Pressure fluctuations in isotropic turbulence," Proc. Camb. Phil. Soc. 47, 359 (1951).

22. S. Chandrasekhar, "The fluctuation of density in isotropic turbulence," Proc. Roy. Soc. London A 210, 18 (1951).

23. J. P. Boon and S. Yip, Molecular Hydrodynamics, McGraw-Hill (1980).

24. A. Yoshizawa, "Simplified statistical approach to complex turbulent flows and ensemble-mean compressible turbulence modeling," Phys. Fluids 7, 3105 (1995).

25. S. L. Woodruff, "A similarity solution for the direct interaction approximation and its relationship to renormalization group analyses of turbulence," Phys. Fluids 6, 3051 (1994).

26. J. D. Fournier, P. L. Sulem, and A. Pouquet, "Infrared properties of forced magnetohydrodynamic turbulence," J. Phys. A 15, 1393 (1982).

27. V. Yakhot and S. A. Orszag, "Renormalization group analysis of turbulence," J. Sci. Comput. 1, 3 (1986). 
28. G. A. Blaisdell, N.N. Mansour, and W.C. Reynolds, "Compressibility effects on the growth and structure of homogeneous turbulent shear flow," J. Fluid Mech. 256, 443 (1993).

29. P. C. Martin, E. Siggia, and H. Rose, "Statistical dynamics of classical systems," Phys. Rev. A 8, 423 (1973). 


\section{REPORT DOCUMENTATION PAGE}

Public reporting burden for this collection of information is estimated to average 1 hour per response, including the time for reviewing instructions, searching existing data sources. gathering and maintaining the data needed. and completing and reviewing the collection of inlormation. Send comments regarding this burden estimate or any olher aspect of this Davis Highway, Suite 1204, Arlingion, VA 22202-4302, and to the Office of Management and Budzet, Paperwork Reduction Projeet (0704-0188). Washington, DC 20503.

\begin{tabular}{|l|c|c|} 
1. AGENCY USE ONLY(Leave blank) & $\begin{array}{c}\text { 2. REPORT DATE } \\
\text { August } 1996\end{array}$ & $\begin{array}{c}\text { 3. REPORT TYPE AND DATES COVERED } \\
\text { Contractor Report }\end{array}$
\end{tabular}

4. TITLE AND SUBTITLE

TRANSPORT COEFFICIENTS IN WEAKLY

COMPRESSIBLE TURBULENCE

5. FUNDING NUMBERS

C NAS1-19480

WU 505-90-52-01

6. AUTHOR(S)

Robert Rubinstein

Gordon Erlebacher

7. PERFORMING ORGANIZATION NAME(S) AND ADDRESS(ES)

Institute for Computer Applications in Science and Engineering

Mail Stop 132C, NASA Langley Research Center

Hampton, VA 23681-0001

8. PERFORMING ORGANIZATION

REPORT NUMBER

ICASE Report No. 96-52

9. SPONSORING/MONITORING AGENCY NAME(S) AND ADDRESS(ES)

National Aeronautics and Space Administration

Langley Research Center

Hampton, VA 23681-0001

10. SPONSORING/MONITORING AGENCY REPORT NUMBER

NASA CR-201595

ICASE Report No. 96-52

\section{SUPPLEMENTARY NOTES}

Langley Technical Monitor: Dennis M. Bushnell

Final Report

Submitted to Physics of Fluids.

\begin{tabular}{l|l} 
12a. DISTRIBUTION/AVAILABILITY STATEMENT & 12b. DISTRIBUTION CODE
\end{tabular}

Unclassified-Unlimited

Subject Category 34

13. ABSTRACT (Maximum 200 words)

A theory of transport coefficients in weakly compressible turbulence is derived by applying Yoshizawa's two-scale direct interaction approximation to the compressible equations of motion linearized about a state of incompressible turbulence. The result is a generalization of the eddy viscosity representation of incompressible turbulence. In addition to the usual incompressible eddy viscosity, the calculation generates eddy diffusivities for entropy and pressure, and an effective bulk viscosity acting on the mean flow. The compressible fluctuations also generate an effective turbulent mean pressure and corrections to the speed of sound. Finally, a prediction unique to Yoshizawa's two-scale approximation is that terms containing gradients of incompressible turbulence quantities also appear in the mean flow equations. The form these terms take is described.

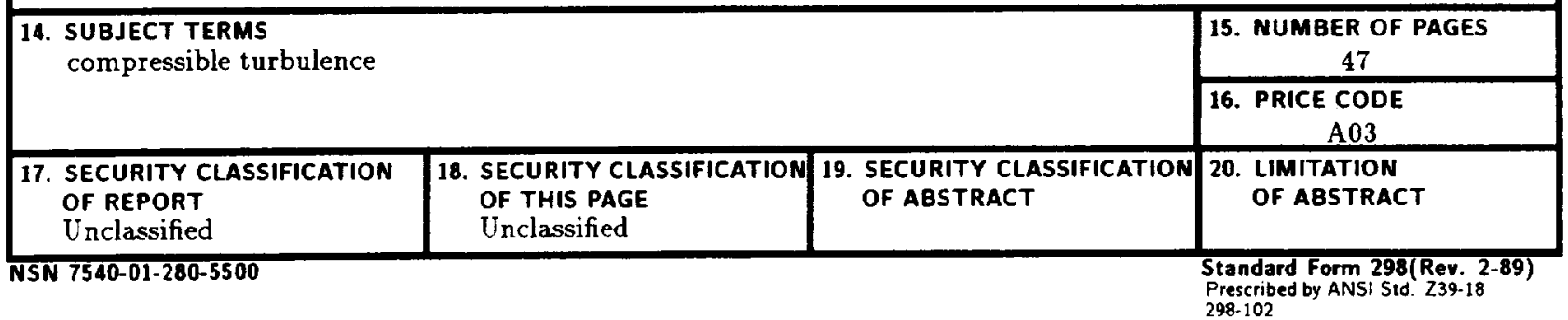

